\title{
Late Prenatal Immune Activation in Mice Leads to Behavioral and Neurochemical Abnormalities Relevant to the Negative Symptoms of Schizophrenia
}

\author{
Byron KY Bitanihirwe', Daria Peleg-Raibstein', Forouhar Mouttet', Joram Feldon' and Urs Meyer*,' \\ 'Department of Biology, Laboratory of Behavioural Neurobiology, Swiss Federal Institute of Technology (ETH) Zurich, Schwerzenbach, Switzerland
}

\begin{abstract}
Based on the human epidemiological association between prenatal infection and higher risk of schizophrenia, a number of animal models have been established to explore the long-term brain and behavioral consequences of prenatal immune challenge. Accumulating evidence suggests that the vulnerability to specific forms of schizophrenia-related abnormalities is critically influenced by the precise timing of the prenatal immunological insult. In the present study, we tested the hypothesis whether late prenatal immune challenge in mice may induce long-term behavioral and neurochemical dysfunctions primarily associated with the negative symptoms of schizophrenia. We found that prenatal exposure to the viral mimic polyriboinosinic-polyribocytidilic acid (Poly-l:C; $5 \mathrm{mg} / \mathrm{kg}$, i.v.) on gestation day (GD) 17 led to significant deficits in social interaction, anhedonic behavior, and alterations in the locomotor and stereotyped behavioral responses to acute apomorphine (APO) treatment in both male and female offspring. In addition, male but not female offspring born to immune challenged mothers displayed behavioral/cognitive inflexibility as indexed by the presence of an abnormally enhanced latent inhibition (LI) effect. Prenatal immune activation in late gestation also led to numerous, partly sex-specific changes in basal neurotransmitter levels, including reduced dopamine (DA) and glutamate contents in the prefrontal cortex and hippocampus, as well as reduced $\gamma$-aminobutyric acid (GABA) and glycine contents in the hippocampus and prefrontal cortex, respectively. The constellation of behavioral and neurochemical abnormalities emerging after late prenatal Poly-l:C exposure in mice leads us to conclude that this immune-based experimental model provides a powerful neurodevelopmental animal model especially for (but not limited to) the negative symptoms of schizophrenia.
\end{abstract}

Neuropsychopharmacology (2010) 35, 2462-2478; doi:I0.1038/npp.2010.129; published online 25 August 2010

Keywords: anhedonia; behavioral/cognitive flexibility; dopamine; glutamate; prefrontal cortex; social interaction

\section{INTRODUCTION}

Human epidemiological studies have repeatedly demonstrated that the risk of developing schizophrenia and related disorders is significantly enhanced following prenatal exposure to infection (Brown, 2006; Brown and Derkits, 2010; Fatemi, 2005; Patterson, 2007). This has motivated the establishment of several rodent models of prenatal infection and/or immune activation, which aim at exploring this epidemiological association on experimental grounds (for a recent review, see Meyer et al, 2009a,b; Meyer and Feldon, 2009, 2010; Patterson, 2009). One such model is based on prenatal exposure to the viral mimic polyriboinosinic-polyribocytidilic acid

*Correspondence: Dr U Meyer, Department of Biology, Laboratory of Behavioural Neurobiology, Swiss Federal Institute of Technology (ETH) Zurich, Schorenstrasse 16, Schwerzenbach 8603, Switzerland, Tel: +41 44655 7403, Fax: +4I 44655 7203, E-mail: urmeyer@ethz.ch or meyer@behav.biol.ethz.ch

Received 23 March 2010; revised 8 July 2010; accepted 23 July 2010
(Poly-I:C) in rats or mice. Poly-I:C is a synthetic analog of double-stranded RNA, which elicits a cytokine-associated viral-like acute phase response in mammalian organisms (Kimura et al, 1994; Traynor et al, 2004). Numerous experimental investigations have provided robust evidence for the emergence of long-term functional and structural brain abnormalities following prenatal exposure to Poly-I:Cinduced immune challenge in both rats and mice (reviewed in Meyer et al, 2009a, b; Meyer and Feldon, 2009, 2010; Patterson, 2009). Importantly, many of the prenatal PolyI:C-induced behavioral, cognitive, and pharmacological dysfunctions in adult offspring are directly implicated in schizophrenia and other psychosis-related disorders, including abnormalities in sensorimotor gating, selective attention, working memory, and sensitivity to psychostimulant drugs. At least some of these functional abnormalities can be normalized by acute and/or chronic antipsychotic drug treatment (Meyer et al, 2010; Ozawa et al, 2006; Shi et al, 2003; Zuckerman et al, 2003; Zuckerman and Weiner, 2005) and are dependent on 
post-pubertal maturational processes (Meyer et al, 2006c, 2008b; Ozawa et al, 2006; Vuillermot et al, 2010; Wolff and Bilkey, 2008; Zuckerman et al, 2003). Taken together, the prenatal Poly-I:C model in rats and mice is characterized by a high level of face, construct, and predictive validity for schizophrenia-like psychopathology, and it successfully mimics the developmental delay of symptom onset (Fatemi and Folsom, 2009; Meyer and Feldon, 2010; Rapoport et al, 2005; Weinberger, 1987).

Using the Poly-I:C model of prenatal immune activation in mice, we have recently shown that the vulnerability to specific forms of schizophrenia-related functional and structural abnormalities is critically influenced by the precise timing of the prenatal immunological challenge (Li et al, 2009; Meyer et al, 2006a,b, 2007, 2008b). For example, abnormalities in selective attention in the form of latent inhibition (LI) disruption (Meyer et al, 2006a) and sensorimotor gating impairment in the form of reduced prepulse inhibition (Li et al, 2009; Meyer et al, 2008b) are part of a symptom cluster that are characteristic for prenatal Poly-I:C-induced immune activation in early/middle gestation. On the other hand, emergence of perseverative behavior in the form of impaired discrimination reversal learning (Meyer et al, 2006b), spatial working memory deficits under low storage load in the temporal domain (Meyer et al, 2008b), and marked potentiation of the sensitivity to $\mathrm{N}$-methyl-D-aspartate (NMDA)-receptor antagonism by acute dizocilpine (MK-801) treatment (Meyer et al, 2008b) are more readily seen following prenatal Poly-I:C exposure in late gestation compared with identical prenatal immune challenge in early/middle gestation. Based on this, it has been suggested that late prenatal immune activation in mice may mimic some critical functional abnormalities relevant to the negative symptoms of schizophrenia (Sullivan et al, 2006; Meyer et al, 2009b). However, experimental support for this hypothesis is relatively sparse thus far, and the extent to which late prenatal immune activation may induce long-term behavioral abnormalities relevant to the negative symptoms of schizophrenia clearly warrants further exploration.

Therefore, the present study tested the hypothesis that prenatal Poly-I:C-induced immune activation in late gestation may lead to multiple behavioral abnormalities relevant to the negative symptoms of schizophrenia. These symptoms refer to features that are normally present in healthy subjects but are reduced or absent as a result of the disease process (Crow, 1980). Besides others, negative symptoms include social interaction deficits, presence of repetitive stereotyped behaviors, anhedonia, and behavioral and/or cognitive inflexibility (Blanchard and Cohen, 2006; Crider, 1997; Crow, 1980; Foussias and Remington, 2010; McGlashan and Fenton, 1992; Morrens et al, 2006). In keeping with our working hypothesis, our phenotypic characterization of the effects of late prenatal immune challenge thus comprised tests which have been designed to assess such behavioral functions in translational rodent models (Arguello and Gogos, 2006; Crawley, 2007; Meyer and Feldon, 2010). In addition to the functional phenotyping, we also explored the neurochemical correlates of behavioral dysfunctions following prenatal immune challenge. This was achieved by means of neurochemical analyses of central monoamines and their metabolites, as well as of excitatory and inhibitory amino acids in postmortem brain tissue of prenatally immune challenged and control offspring.

\section{MATERIALS AND METHODS}

\section{Animals}

C57BL/6 mice were used throughout the study. Female and male breeders were obtained from our in-house specific pathogen free colony at the age of 10-14 weeks. Breeding began after 2 weeks of acclimatization to the new animal holding room, which was a temperature and humidity controlled $\left(21 \pm 1^{\circ} \mathrm{C}, 55 \pm 5 \%\right)$ holding facility under a reversed light-dark cycle (lights off: 08:00-20:00 hours). All animals had ad libitum access to food (Kliba 3430, Klibamühlen, Kaiseraugst, Switzerland) and water. All procedures described in the present study had been previously approved by the Cantonal Veterinarian's Office of Zurich and are in agreement with the principles of laboratory animal care in the Guide for the Care and Use of Laboratory Animals (National Institutes of Health Publication No. 86-23, revised 1985). All efforts were made to minimize the number of animals used and their suffering.

\section{Maternal Immune Activation in Late Gestation}

For the purpose of the maternal immunological manipulation in late gestation, female mice were subjected to a timed mating procedure as described previously (Meyer et al, 2005). Pregnant dams on gestation day (GD) 17 received either a single injection of Poly-I:C (potassium salt; Sigma-Aldrich, Buchs, St Gallen, Switzerland) or vehicle. Poly-I:C $(5 \mathrm{mg} / \mathrm{kg})$ was dissolved in sterile pyrogen-free $0.9 \% \mathrm{NaCl}$ (vehicle) solution to yield a final concentration of $1 \mathrm{mg} / \mathrm{ml}$ and was administered via the intravenous (i.v.) route at the tail vein under mild physical constraint. The dose of Poly-I:C was chosen based on our previous studies in C57BL/6 mice (Meyer et al, 2006a, b, 2008b). All solutions were freshly prepared on the day of administration and were injected at a volume of $5 \mathrm{ml} / \mathrm{kg}$.

The selected gestational window (ie, GD 17) in mice corresponds roughly to the middle-to-late second trimester of human pregnancy, with respect to developmental biology and percentage of gestation from mice to human (Clancy et al, 2001; Clancy et al, 2007). This gestational stage is identical to the one selected in our previous experimental studies examining the impact of the precise timing of prenatal immune activation on postnatal brain dysfunctions (Li et al, 2009; Meyer et al, 2006a, b, 2008c).

\section{Phenotypic Characterization of Offspring}

All offspring were weaned and sexed on postnatal day (PND) 21. Littermates of the same sex were caged separately. All animals were maintained under ad libitum food and water, and were kept in a temperature and humidity controlled animal vivarium under a $12: 12 \mathrm{~h}$ reversed light-dark cycle as described above.

Offspring from multiple independent litters (eight PolyI:C, eight saline) were randomly selected for the behavioral and pharmacological tests. Both male and female offspring were included in all the tests described below to assess 
potential sex-dependent effects of the prenatal immunological manipulation. A first cohort of Poly-I:C and control offspring $(N=16$ (eight males, eight females) in each experimental group) was subjected to behavioral testing in the following test order: (1) social interaction test, (2) sucrose preference test, and (3) LI test (see below). All animals were allowed a 1-week resting period between each test. The first cohort of Poly-I:C and control offspring was then used for postmortem neurochemical analyses (see below) following a resting period of 4 weeks upon conclusion of the LI test. A second cohort of Poly-I:C and control offspring $(N=12$ (six males, six females) in each experimental group) was used for the assessment of basal and APO-induced stereotypies (see below). All behavioral testings commenced when the offspring reached the adult stage of development (ie, PND 80 onwards).

\section{Social Interaction Test}

Social behavior is commonly referred to as the behavior that occurs in a social context and results from the interaction between and among individuals (of the same species). As mice (like most other rodents) are highly social animals, social interaction can be efficiently studied under experimental conditions (Crawley, 2007). Social interaction was assessed by analyzing the relative exploration time between an unfamiliar congenic mouse and an inanimate dummy object. A detailed description of the test apparatus and procedures used in the social interaction test can be obtained in the Supplementary Materials and Methods.

\section{Sucrose Preference Test}

Anhedonic behavior was assessed by a standard sucrose preference test using methods adapted from previous studies (Hayward et al, 2006; Slattery et al, 2007). The sucrose preference test is based on the observation that rodents typically show a preference for a sweet sucrose solution when presented with a free choice between the sucrose solution and water. A reduction in this preference is commonly considered as an indication of anhedonia (Cryan and Mombereau, 2004; Hayward et al, 2006; Slattery et $a l, 2007)$ and conversely an increase may suggest hyperhedonia. A detailed description of the apparatus and experimental procedures used can be obtained in the Supplementary Materials and Methods. Sucrose preference was indexed by a percentage score (sucrose consumption/ (total liquid consumption) $\times 100 \%$ ) obtained on two consecutive test days (Supplementary Materials and Methods). In addition, we also recorded and analyzed total fluid consumption during the habituation and test phases (Supplementary Materials and Methods) to compare the general fluid intake between the two prenatal treatment groups.

\section{Test}

Behavioral/cognitive inflexibility was studied by assessing the presence of LI persistence. LI is a selective learning procedure, in which previous repeated pre-exposures to the to-be-conditioned stimulus (CS) alters the subsequent development of the conditioned response (CR) following explicit parings between the same CS and an unconditioned stimulus (US; Weiner, 2003). A significant LI effect (ie, impaired conditioning following repeated inconsequential pre-exposures to the CS) is typically manifest when the organism is subjected to a high number of CS-preexposures (CS-PE) before CS-US conditioning (Weiner, 2003; Weiner and Arad, 2009). In contrast, presentation of a low number of CS-alone stimuli during the pre-exposure phase readily prevents the expression of LI in normal subjects (Weiner, 2003; Weiner and Arad, 2009). However, organisms which tend to show behavioral and/or cognitive inflexibility are expected to express the LI effect under parametric conditions that are insufficient for control animals to display LI (Weiner, 2003; Weiner and Arad, 2009).

LI persistence was studied in a two-way active avoidance procedure using four identical two-way shuttle boxes. The test apparatus used has been fully described before (Meyer et al, 2006c). A white noise stimulus served as the CS and electric foot shock served as the US. To facilitate the anticipated expression of LI persistence in prenatally immune challenged offspring relative to control offspring, we used an LI procedure, which has been shown in preliminary studies to result in minimal or no LI in adult control C57BL/6 mice (Meyer, Feldon: unpublished observations). The test procedures consisted of two phases: pre-exposure and conditioning, conducted $24 \mathrm{~h}$ apart. Equal numbers of offspring from both prenatal treatment (PolyI:C or vehicle) conditions were allocated to one of the two conditions: CS-PE and non-pre-exposure (NPE). In the pre-exposure phase, CS-PE subjects were presented with 40 pre-exposures to a 5-s white noise CS according to a random interstimulus interval schedule, whereas NPE subjects were confined to the chamber for an equivalent period of time without any stimulus presentation. On the conditioning day, the subjects were returned to the same shuttle boxes and received a total of 100 avoidance trials. A detailed description of the test procedures can be found in the Supplementary Materials and Methods.

To index conditioned avoidance learning, the mean response latency performed on successive 10-trial blocks was analyzed (Supplementary Materials and Methods). In addition, the mean numbers of spontaneous shuttles during the inter-trial intervals (ITIs) were recorded and analyzed to account for basal locomotor activity during the conditioning phase.

\section{Basal and APO-Induced Stereotyped Behavior}

Behavioral stereotypy is defined as uniform, repetitive, and compulsive actions within a restricted pattern, which often have no obvious goals or end points. Acute treatment with the mixed DA D1/D2 receptor agonist APO is well known to induce a dose-dependent enhancement of stereotyped behaviors in mice, including repetitive climbing, wallleaning, sniffing, and gnawing (Protais et al, 1976; Cabib and Puglisi-Allegra, 1985; Tirelli and Witkin, 1994). Acute APO treatment can thus be used to probe the sensitivity to drug-induced stereotyped behavioral responses.

Basal and APO-induced stereotyped behaviors were assessed by analyzing horizontal and vertical movements and behaviors in a wire mesh cylinder following vehicle 
(vitamin C solution (VitC)) or APO ( $5 \mathrm{mg} / \mathrm{kg}$, s.c.) administration. A detailed description of the test apparatus and experimental procedures used can be found in the Supplementary Materials and Methods.

\section{Postmortem Neurochemistry}

Levels of monoamines (noradrenaline (NA), DA, serotonin (5-hydroxytryptamine (5-HT))) and their metabolites (dihydroxyphenylacetic acid (DOPAC), homovanillic acid (HVA), 5-hydroxyindoleacetic acid (5-HIAA), 3-methoxytyramine (3-MT)), amino acids ( $\gamma$-aminobutyric acid (GABA), glutamate, aspartate, glycine), and the sulfonic amino acid taurine were determined using high-performance liquid chromatography (HPLC) according to procedures established before (Peleg-Raibstein et al, 2005; Peleg-Raibstein et al, 2008) and are described in detail in the Supplementary Materials and Methods. The rationale of measuring brain levels of monoamines, glutamate, aspartate, and glycine was primarily because these neurotransmitters have all been implicated in the modulation of behavioral/cognitive flexibility (Dalley et al, 2004; Errico et al, 2008; Singer et al, 2009; Weiner and Arad, 2009), as well as in the regulation of hedonic and social behavior (Berridge and Robinson, 1998; Fernandez Espejo, 2003). The rationale of including measurements of GABA and taurine was to extend our previous findings of prenatal Poly-I:C-induced neuroanatomical alterations in GABA-related markers (Meyer et al, 2008c) to measurements at the neurochemical level.

Following decapitation and dissection of the brain, coronal sections were prepared using razorblade cuts along the following coordinates with respect to bregma: anteriorposterior +2.3 to +1.3 , +1.3 to $+0.3,-0.1$ to $-0.6,-1.2$ to -2.2 , and -2.8 to -3.8 . Discrete brain regions were then collected using a micropunch needle ( $1 \mathrm{~mm}$ in diameter) generating micropunches for the medial prefrontal cortex (mPFC), amygdala (AMY), caudate putamen $(\mathrm{CPu})$, nucleus accumbens (NAc), dorsal hippocampus (dHPC), and ventral hippocampus (vHPC; for a detailed description, see Supplementary Materials and Methods). Monoamines and amino acids were determined using amperometric electrochemical and fluorescence detectors, respectively (see Supplementary Materials and Methods for detailed information).

\section{Statistical Analyses}

All statistical analyses were conducted using the Statistical Package for the Social Sciences (SPSS) software (version 13.0) implemented on a personal computer running the Windows XP operating system. Statistical significance was set at $P<0.05$.

All behavioral and pharmacological data were analyzed using parametric analysis of variance (ANOVA) followed by Fisher's least significant difference post hoc group comparisons or restricted ANOVA whenever appropriate. In the social interaction test, exploration time was expressed as a function of 1 -min bins and analyzed using a $2 \times 2 \times 2 \times 5$ (prenatal treatment $\times$ sex $\times$ object $\times 1$-min bin) repeated measures ANOVA (RM-ANOVA). Additional $2 \times 2 \times 5$ (sex $\times$ object $\times$ 1-min bin) RM-ANOVAs of exploration time restricted to control and Poly-I:C offspring were further conducted to confirm the presence or absence of a significant preference towards the live unfamiliar mouse $v s$ the inanimate dummy object. In the sucrose preference test, baseline fluid consumption during the initial 72-h habituation phase was evaluated using a $2 \times 2 \times 3$ (prenatal treatment $\times$ sex $\times$ days) RMANOVA of water consumption. Sucrose preference was then assessed using a $2 \times 2 \times 2$ (prenatal treatment $\times \operatorname{sex} \times$ days) RM-ANOVA of percentage sucrose intake during the subsequent 48 -h test period. Total fluid intake during the 48 -h test period was also analyzed using a $2 \times 2 \times 2$ (prenatal treatment $\times$ sex $\times$ days) RM-ANOVA. In the two-way active avoidance LI paradigm, conditioned avoidance learning (indexed by the response latency) was expressed as a function of 10-trials blocks and analyzed using a $2 \times 2 \times 2 \times 10$ (prenatal treatment $\times$ sex $\times$ pre-exposure $\times 10$-trials block) RM-ANOVA, followed by a posteriori $2 \times 10$ (pre-exposure $\times 10$-trials block) $\mathrm{RM}$-ANOVA restricted to each prenatal treatment condition and/or sex, if appropriate. In the APO sensitivity test, locomotor activity (indexed by the horizontal distance traveled in wire mesh cylinder) was expressed as a function of 5-min bins and analyzed using a $2 \times 2 \times 6$ (prenatal treatment $\times$ sex $\times$ bins) RM-ANOVA for the VitC treatment phase, and by a $2 \times 2 \times 12$ (prenatal treatment $\times \operatorname{sex} \times$ bins) for the APO treatment phase. Behavioral measures obtained in the analysis of stereotyped responses to VitC and APO treatment (ie, scores for climbing, leaning, and sniffing behavior) were first subjected to squareroot transformation to better conform the data to the normal distribution assumption of parametric ANOVA. These measures were then analyzed using a $2 \times 2 \times 5$ (prenatal treatment $\times$ sex $\times$ sampling interval) RM-ANOVA following VitC treatment, and by a $2 \times 2 \times 11$ (prenatal treatment $\times$ sex $\times$ sampling interval) RM-ANOVA following APO treatment.

Each neurotransmitter, metabolite or metabolite/neurotransmitter ratio of interest was first analyzed using a $2 \times$ $2 \times 6$ (prenatal treatment $\times$ sex $\times$ brain area) RM-ANOVA. The within-subjects factor of brain area was included to explore whether the anticipated effects of prenatal immune challenge on specific neurotransmitters, metabolites or metabolite/neurotransmitter ratios would be dependent on the precise brain area investigated. Subsequent $2 \times 2$ (prenatal treatment $\times$ sex) ANOVAs restricted to individual brain areas were conducted if the initial RM-ANOVA revealed a significant interaction between prenatal treatment and brain area. If the initial RM-ANOVA revealed a significant threeway interaction between prenatal treatment, sex, and brain area, the effects of prenatal immune activation on neurotransmitter, metabolite or metabolite/neurotransmitter ratios in individual brain areas were investigated separately in male and female animals using ANOVA restricted to sex. Finally, if the initial $2 \times 2 \times 6$ (prenatal treatment $\times \operatorname{sex} \times$ brain area) RM-ANOVA failed to detect any significant interactions involving the between-subjects factor of prenatal treatment, no further restricted ANOVAs were conducted.

\section{RESULTS}

\section{Late Prenatal Immune Challenge Leads to Reduced Social Interaction}

The relative exploration time between an unfamiliar congenic mouse and an inanimate dummy object was used to assess 

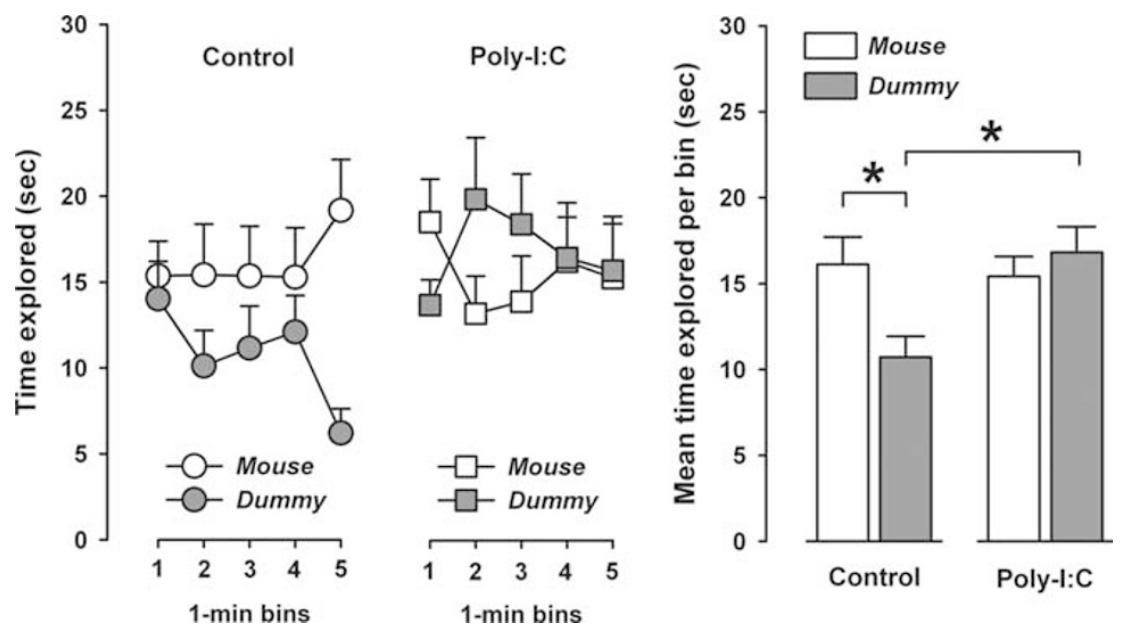

Figure I Social interaction deficits following late prenatal immune challenge. Social interaction was assessed by analyzing the relative exploration time between an unfamiliar congenic mouse ('mouse') and an inanimate dummy object ('dummy'). The line plots depict the relative exploration time as a function of successive I-min bins, and the bar plot shows the mean exploration time per bin. Control offspring born to vehicle-treated mothers displayed a significant preference towards the unfamiliar congenic mouse, indicating intact social interaction. On the other hand, offspring exposed to prenatal Poly-l:C treatment in late gestation displayed no such preference. ${ }^{*} P<0.05$, based on restricted ANOVAs. All values are means \pm SEM.

social interaction in adult control and Poly-I:C offspring. The relative exploration time was recorded during a 5-min test period and analyzed as a function of 1-min bins. As shown in Figure 1, control offspring born to vehicle-treated mothers displayed a clear preference towards the unfamiliar mouse, indicating intact social interaction. On the other hand, offspring exposed to prenatal Poly-I:C treatment in late gestation displayed no such preference (Figure 1). The PolyI:C-induced disruption of social interaction was evident in both male and female offspring.

Statistical support for these impressions was obtained by RM-ANOVA of exploration time, which yielded a significant main effect of treatment $(\mathrm{F}(1,28)=4.56, P<0.05)$ and its interaction with object $(\mathrm{F}(1,28)=6.07, P<0.05)$. Additional RM-ANOVA of exploration time restricted to control and Poly-I:C offspring was then conducted to further confirm the presence or absence of a significant preference towards the live unfamiliar mouse $v s$ the inanimate dummy object. By revealing a significant main effect of object $(\mathrm{F}(1,14)=8.23$, $P<0.05$ ), the RM-ANOVA restricted to control offspring confirmed a significant preference towards the live mouse. In contrast, the main effect of object (or its interaction with bins) was far from significant in the restricted RM-ANOVA of exploration time in Poly-I:C offspring $(\mathrm{F}<0.5)$. Additional RM-ANOVAs of exploration time restricted to the two objects (ie, live mouse and inanimate dummy object) were also conducted. The analysis restricted to the inanimate dummy object revealed a significant main effect of prenatal treatment $(\mathrm{F}(1,28)=9.28, P<0.05)$, reflecting the significant increase in dummy object exploration exhibited by Poly-I:C offspring compared with control offspring (Figure 1). The RM-ANOVA restricted to the live mouse did not reveal any significant main effects or interactions.

\section{Late Prenatal Immune Challenge Leads to Anhedonic Behavior in the Sucrose Preference Test}

Prenatal Poly-I:C-induced immune activation on GD17 did not significantly affect basal water intake during the habituation phase of the sucrose preference test. RMANOVA of water consumption during the initial 72-h habituation phase did not reveal any significant main effects or interactions. The overall daily mean \pm SEM of water intake during this phase was $7.67 \pm 0.24 \mathrm{ml}$. Similarly, RMANOVA of total fluid (water and sucrose solution) consumption during the subsequent 48 -h test period did not reveal any significant main effects or interactions, indicating that the amount of total fluid consumed during the two test days was highly comparable between the two prenatal treatment groups and sex (Figure $2 \mathrm{~b}$ ). The overall mean \pm SEM of total fluid (water and sucrose solution) intake during the test phase was $6.36 \pm 0.18 \mathrm{ml}$.

However, prenatal Poly-I:C exposure significantly reduced sucrose preference (as indexed by the percentage score of sucrose consumption) compared with prenatal control treatment (Figure 2a). This specific effect of the prenatal immunological manipulation was equally seen at each of the two test days and emerged in both male and female offspring. Statistical support for these impressions was yielded by the RM-ANOVA of percentage sucrose intake, which revealed a significant main effect of prenatal treatment $(\mathrm{F}(1,28)=7.89, P<0.01)$. No other main effects or interactions reached statistical significance.

\section{Late Prenatal Immune Challenge Leads to Abnormally Enhanced LI}

Conditioned avoidance learning was assessed by analyzing the mean response latency performed on successive 10trials blocks. Acquisition of the $\mathrm{CR}$ was evident by a significant decrease in response latency as a function of blocks, leading to a significant main effect of blocks $(\mathrm{F}(9,216)=35.03, P<0.001)$ in the RM-ANOVA of response latency. As expected, pre-exposures to a low number of the white-noise CS did not induce a LI effect (ie, a decrease in conditioned responding following repeated CS-PE before conditioning) in offspring born to control mothers (Figure 3). Hence, the response latency between control 

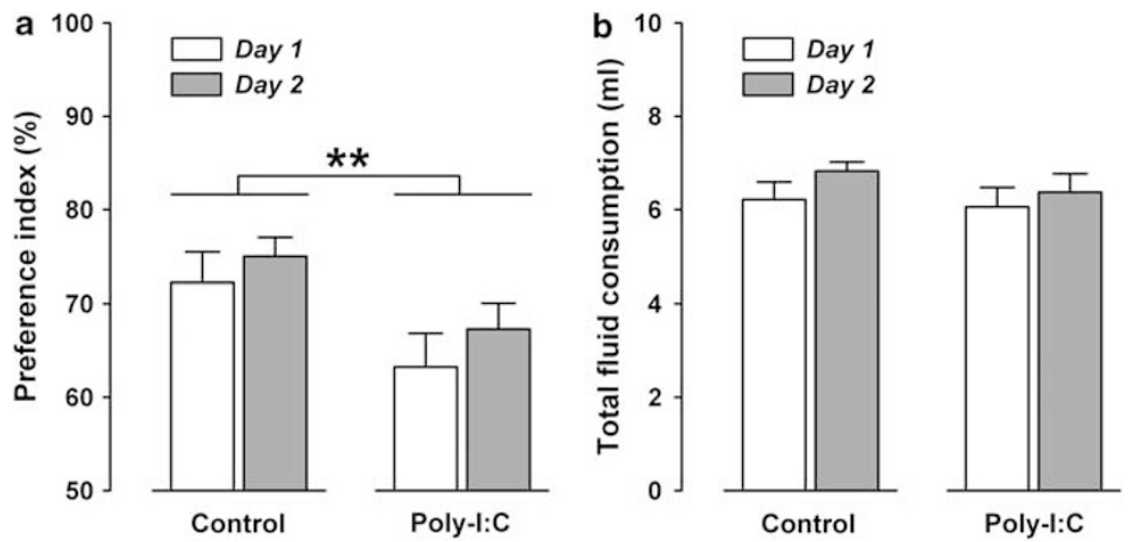

Figure 2 Presence of anhedonic behavior following late prenatal immune challenge. Anhedonic behavior was assessed using a 2-day (48h) sucrose preference test, in which animals had free choice between regular tap water and a $0.5 \%$ sucrose solution. (a) The graph depicts sucrose preference as indexed by a percentage score (sucrose consumption/(total liquid consumption) $\times 100 \%$ ) obtained on two consecutive test days (day I and 2). Adult offspring exposed to prenatal Poly-l:C treatment in late gestation displayed a significant reduction in the sucrose preference index compared with adult control offspring. $* * P<0.0$ I, signifies the main effect of prenatal treatment in the corresponding RM-ANOVA. (b) The graph shows the total fluid (water + sucrose solution) consumption on the two consecutive test days. Prenatal Poly-l:C treatment did not affect total fluid intake across the two test days. All values are means \pm SEM.

MALES

FEMALES
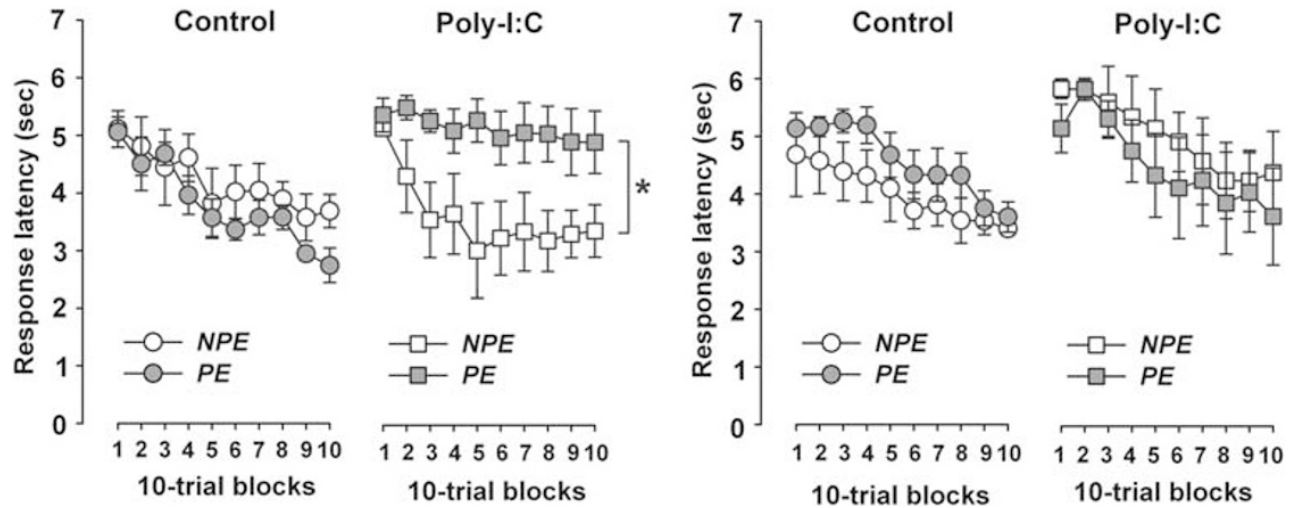

Figure 3 Presence of abnormally enhanced LI following late prenatal immune challenge. The LI effect was studied in a two-way active avoidance procedure, in which pre-exposed (PE) subjects were presented with a low number of the CS before conditioning, whereas NPE subjects were not pre-exposed to the CS. The graph depicts the response latency as a function of blocks of 10 trials in male and female offspring. Male but not female offspring exposed to prenatal Poly-l:C treatment in late gestation displayed a significant LI effect (ie, reduced response latency in PE relative to NPE subjects) under parametric conditions, in which control offspring did not display LI. $* P<0.05$, signifies the significant main effect of pre-exposure in the corresponding ANOVA restricted to male Poly-l:C offspring. All values are means \pm SEM.

NPE and PE subjects was highly comparable (Figure 3 ). However, prenatal Poly-I:C exposure led to a sex-dependent enhancement of the LI effect in male offspring born to PolyI:C-treated mothers (Figure 3). This pattern of results led to a significant three-way interaction between prenatal treatment, sex, and pre-exposure in the RM-ANOVA of response latency $(\mathrm{F}(1,24)=6.72, P<0.05)$. An additional RM-ANOVA restricted to male Poly-I:C subjects provided further statistical support for the presence of significant LI effect by revealing a significant main effect of pre-exposure $(\mathrm{F}(1,6)=5.87, P<0.05)$.

As depicted in Figure 3, the emergence of a significant LI effect in male Poly-I:C offspring was attributable to delayed learning in CS-PE subjects relative to CS-PE control offspring. RM-ANOVA restricted to male CS-PE subjects revealed a significant main effect of prenatal treatment $(\mathrm{F}(1,6)=12.30, P<0.05)$ and a significant two-way interaction $(\mathrm{F}(9,54)=2.72, P<0.05)$. In contrast, RM-ANOVA restricted to male NPE subjects did not provide any evidence for a significant effect of the prenatal manipulation on conditioned learning in NPE subjects.

Locomotor activity as indexed by the spontaneous ITI shuttles during conditioning decreased in all animals as a function of successive 10-trial blocks, leading to a significant main effect of blocks in the RM-ANOVA of ITI shuttles $(F(9,216)=119.11, P<0.001)$. This was not significantly influenced by the prenatal treatment or stimulus pre-exposure conditions. However, female offspring generally displayed a higher number of ITI shuttles compared with male offspring regardless of the prenatal treatment and 
a
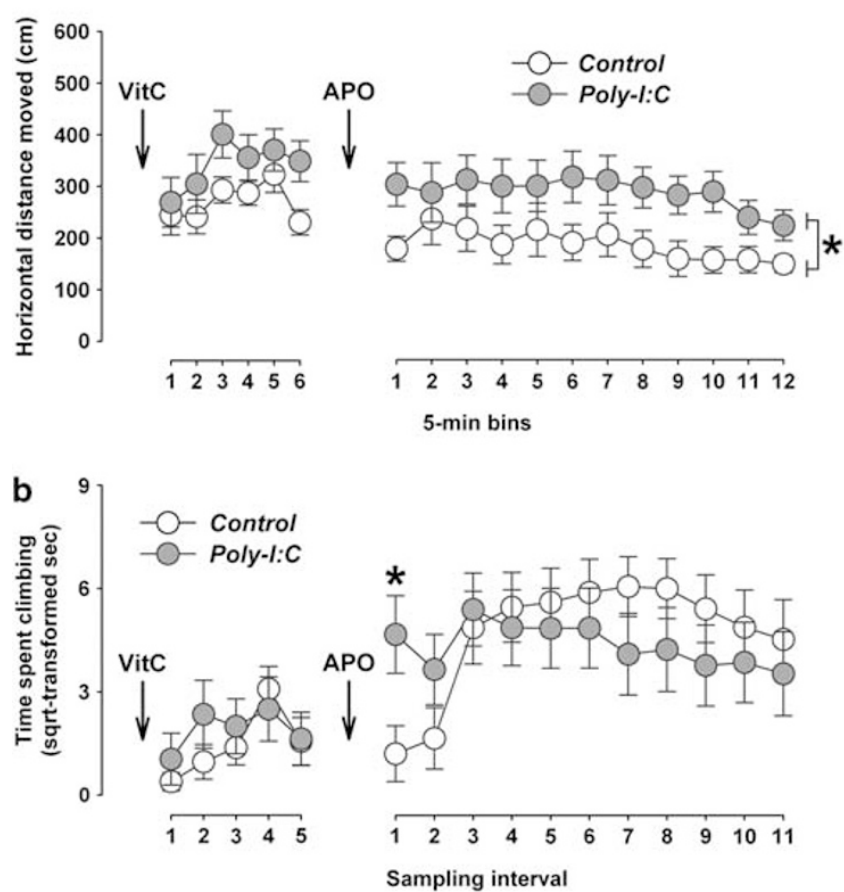

C
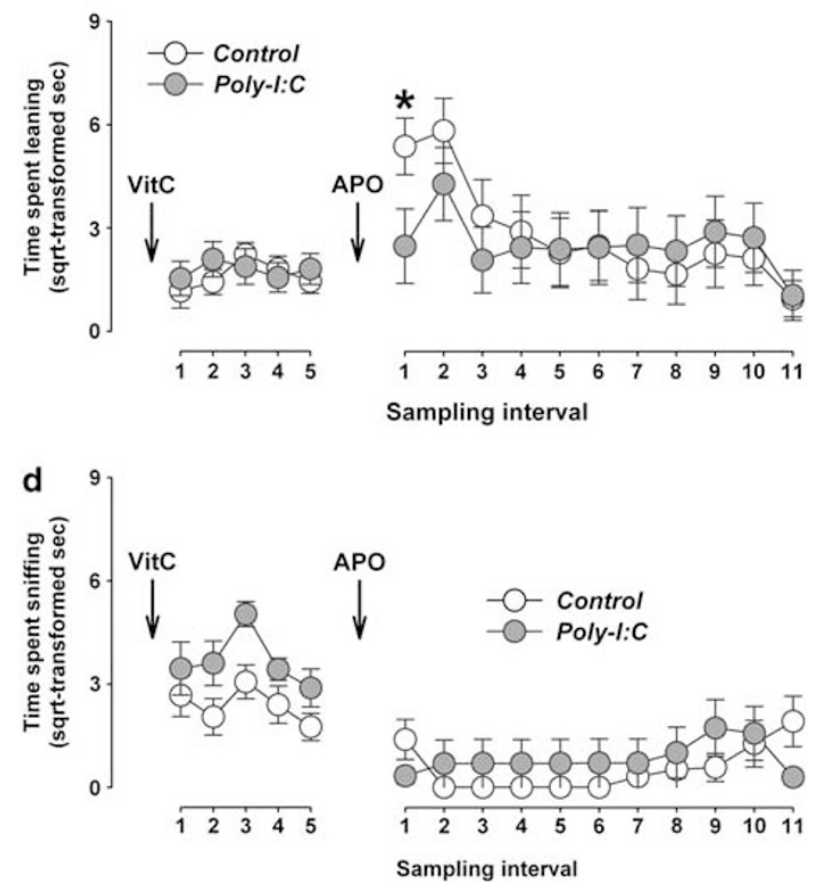

Sampling interval

Figure 4 Late prenatal immune activation leads to altered locomotor and stereotyped behavioral responses to systemic APO treatment. (a) The graph depicts locomotor activity as indexed by the horizontal distance moved following systemic ascorbic acid (VitC, vehicle) treatment and following systemic APO (5 mg/kg, s.c.) treatment. Horizontal distance moved is plotted as a function of 5-min bins. Adult offspring subjected to prenatal Poly-l:C exposure in late gestation displayed an overall significant increase in the horizontal distance moved following APO treatment relative to APO-treated offspring born to control mothers. $* P<0.05$, signifies the significant main effect of prenatal treatment in the corresponding RM-ANOVA. (b) The graph shows the time spent climbing on successive sampling intervals (I-min observation period every $5 \mathrm{~min}$ ) following VitC and APO treatment. Adult Poly-l:C offspring displayed a significant increase in the time spent climbing specifically during the first observation period compared with APO-treated control offspring. $* P<0.05$, signifies the significant main effect of prenatal treatment in the corresponding ANOVA restricted to the first observation period following APO treatment. (c) The graph depicts the time spent leaning on successive sampling intervals (I -min observation period every 5 min) following VitC and APO treatment. Adult PolyI:C offspring displayed a significant decrease in the time spent leaning specifically during the first observation period compared with APO-treated control offspring. $* P<0.05$, signifies the significant main effect of prenatal treatment in the corresponding ANOVA restricted to the first observation period following APO treatment. (d) The graph depicts the time spent sniffing on successive sampling intervals (I-min observation period every 5 min) following VitC and APO treatment. There were no significant group differences in the time spent sniffing neither in the initial VitC phase nor in the subsequent APO phase. All values are means \pm SEM.

stimulus pre-exposure conditions. This led to a significant main effect of sex $(\mathrm{F}(1,24)=8.27, \quad P<0.01)$ and its interaction with 10 -trial blocks $(\mathrm{F}(9,216)=3.93, P<0.01)$. The mean \pm SEM of ITI shuttles per 10-trials block was $1.70 \pm 0.30$ and $0.93 \pm 0.21$ in female and male offspring, respectively.

As our findings of a sex-specific enhancement of the LI effect in male Poly-I:C offspring (Figure 3) were based on a small number of NPE and CS-PE subjects (four male subjects per pre-exposure condition and prenatal treatment group), we re-assessed the effects of prenatal Poly-I:C exposure on LI enhancement in an independent experiment. As outlined in the Supplementary Data, we were able to successfully replicate our initial findings in a second independent cohort of male Poly-I:C and control offspring (Supplementary Data). The successful replication suggests that our initial findings of LI enhancement in male Poly-I:C offspring (Figure 3) can not be accounted for by spurious findings and/or possible litter effects, but rather represents a robust effect of the prenatal treatment on behavioral/ cognitive perseveration in selective associative learning.

\section{Late Prenatal Immune Challenge Leads to Altered Locomotor and Stereotyped Behavioral Responses to Acute APO Treatment}

Horizontal locomotor activity. Figure 4a shows that adult Poly-I:C offspring appeared to display increased levels of horizontal locomotor activity compared with adult control offspring in the initial vehicle (VitC) phase. However, RMANOVA of horizontal locomotor activity following VitC administration failed to attain a significant main effect or interaction involving the between-subjects factor of prenatal treatment. In contrast, a significant difference between Poly-I:C and control offspring was obtained in terms of horizontal locomotor activity following acute APO administration, with APO-treated Poly-I:C offspring displaying a general increase in horizontal locomotor activity compared with APO-treated control offspring (Figure 4a). This effect of prenatal Poly-I:C exposure emerged similarly in male and female offspring, and was statistically supported by the presence of a significant main effect of prenatal treatment $(\mathrm{F}(1,19)=5.80, P<0.05)$ in the RM-ANOVA of horizontal 
locomotor activity following APO administration. Horizontal locomotor activity was generally increased in APOtreated female mice compared with APO-treated male mice regardless of the prenatal treatment conditions. This led to a significant main effect of $\operatorname{sex}(\mathrm{F}(1,19)=4.01, P<0.05)$. However, the between-subjects factor of sex did not interact significantly with the factor of prenatal treatment $(\mathrm{F}<1)$. The mean \pm SEM $(\mathrm{cm})$ of horizontal locomotor activity displayed per 5 -min bin was $281.60 \pm 14.18$ and $193.48 \pm 8.92$ in female and male offspring, respectively.

Climbing. There were no significant differences between Poly-I:C and control offspring in terms of climbing behavior during the initial vehicle phase (Figure $4 \mathrm{~b}$ ). Administration of APO led to a marked increase in climbing behavior (Figure 4b). Most interestingly, Poly-I:C offspring displayed a faster onset of APO-induced enhancement in climbing behavior in comparison with control offspring (Figure 4b). Indeed, although climbing behavior in APOtreated Poly-I:C offspring approached peak levels immediately after APO treatment, climbing behavior in APOtreated control offspring reached peak levels only by $15 \mathrm{~min}$ following the APO injection (Figure 4b). Statistical support for these impressions was obtained by the RM-ANOVA of climbing behavior following APO administration, which yielded a significant main effect of sampling interval $(\mathrm{F}(10,190)=4.63, P<0.001)$ and its interaction with prenatal treatment $(\mathrm{F}(10,190)=3.08, P<0.01)$. Additional ANOVAs restricted to each of the 11 sampling intervals were then performed to further verify the presence or absence of significant group or sex differences in climbing behavior at specific post-injection intervals. By revealing a significant main effect of prenatal treatment $(F(1,19)=7.77$, $P<0.05$ ), the ANOVA restricted to the first sampling interval confirmed that Poly-I:C offspring displayed a faster onset of APO-induced climbing behavior compared with controls (Figure $4 \mathrm{~b}$ ). This time-dependent effect of the PolyI:C treatment emerged in both male and female offspring.

Leaning. In agreement with the outcomes in the analysis of climbing behavior (Figure $4 \mathrm{~b}$ ), leaning behavior was highly comparable between Poly-I:C and control offspring during the initial VitC phase (Figure 4c). Acute APO treatment led to a temporary increase in leaning behavior: It led to an initial increase, which was most pronounced during the first 10 min following APO administration, but subsided later (Figure 4b). This led to a highly significant main effect of sampling interval $(\mathrm{F}(10,190)=5.50, P<0.001)$ in the RMANOVA of leaning behavior following APO administration. Interestingly, the APO-induced temporary increase in leaning behavior appeared to be blunted in Poly-I:C offspring relative controls (Figure $4 \mathrm{~b}$ ), as indicated by the prenatal treatment $\times$ sampling time interaction approaching statistical significance $(\mathrm{F}(10,190)=1.82, P=0.059)$. Additional ANOVAs restricted to each of the 11 sampling intervals confirmed that Poly-I:C offspring displayed reduced leaning behavior relative to controls specifically during the first sampling interval, as supported by the presence of a significant main effect of prenatal treatment $(\mathrm{F}(1,19)=4.89, P<0.05)$ in the ANOVA restricted to the first sampling interval. This specific effect of the Poly-I:C treatment emerged in both male and female offspring.
Sniffing. Figure $4 \mathrm{~d}$ depicts sniffing behavior displayed by Poly-I:C and control offspring during the initial vehicle (VitC) and subsequent APO phase. Prenatal Poly-I:C treatment appeared to increase the amount of sniffing during the VitC phase compared with sniffing displayed by VitC-treated control offspring (Figure 4d). However, the main effect of prenatal treatment in the RM-ANOVA of sniffing following VitC administration only attained statistical trend level $(\mathrm{F}(1,19)=4.31, P=0.0516)$. As depicted in Figure 4d, APO administration led to a noticeable reduction in the amount of sniffing relative to sniffing measured in the initial VitC phase. The APO-induced reduction in sniffing displayed by Poly-I:C offspring appeared to be maximal at the first and last sampling interval, whereas the APOinduced reduction in sniffing displayed by control offspring was maximal between sampling intervals 2 and 6 (Figure 4d). These patterns of results led to a significant main effect of sampling interval $(\mathrm{F}(10,190)=2.78, P<0.01)$ and its interaction with prenatal treatment $(\mathrm{F}(10,190)=$ $2.60, P<0.01)$.

Grooming and biting/gnawing. The amount of grooming and biting/gnawing behavior in Poly-I:C and control offspring was generally very low, both following VitC and APO administration; no significant group differences were revealed in the corresponding statistical analyses (data not shown).

\section{Region- and Sex-Specific Effects of Late Prenatal Immune Challenge on Brain Monoamine Contents}

NA. Prenatal Poly-I:C exposure did not significantly affect levels of NA in the brain (data not shown). RM-ANOVA of NA contents did not reveal a significant main effect or interactions involving the between-subjects factor or prenatal treatment.

$D A$. Prenatal immune activation significantly influenced brain levels of DA in a region-specific manner, as indicated by the presence of significant main effect of prenatal treatment $(\mathrm{F}(1,21)=5.11, P<0.05)$ and its interaction with brain region $(\mathrm{F}(5,105)=2.60, P<0.05)$ in the initial $\mathrm{RM}$ ANOVA. Subsequent ANOVAs restricted to individual brain areas showed that prenatal Poly-I:C treatment led to a significant reduction in DA contents specifically in the mPFC (main effect of prenatal treatment: $\mathrm{F}(1,27)=6.68$, $P<0.05$ ) and vHPC (main effect of prenatal treatment: $\mathrm{F}(1,25)=7.25, P<0.05$; Figure 5a). The effects of the prenatal Poly-I:C treatment were apparent in both male and female offspring.

Serotonin (5-HT). Prenatal Poly-I:C exposure led to sexand region-specific changes in 5-HT, as supported by the presence of a significant three-way interaction between prenatal treatment, sex, and brain area $(F(5,115)=2.52$, $P<0.05)$. By revealing a significant main effect of prenatal treatment $(\mathrm{F}(5,115)=2.52, P<0.05)$ and its interaction with brain areas $(\mathrm{F}(5,115)=2.52, P<0.05)$, the subsequent $\mathrm{RM}$ ANOVA restricted to female subjects indicated that prenatal Poly-I:C treatment significantly influenced 5-HT levels in females. Additional ANOVAs restricted to individual brain areas confirmed that female offspring born to Poly-I: 
a

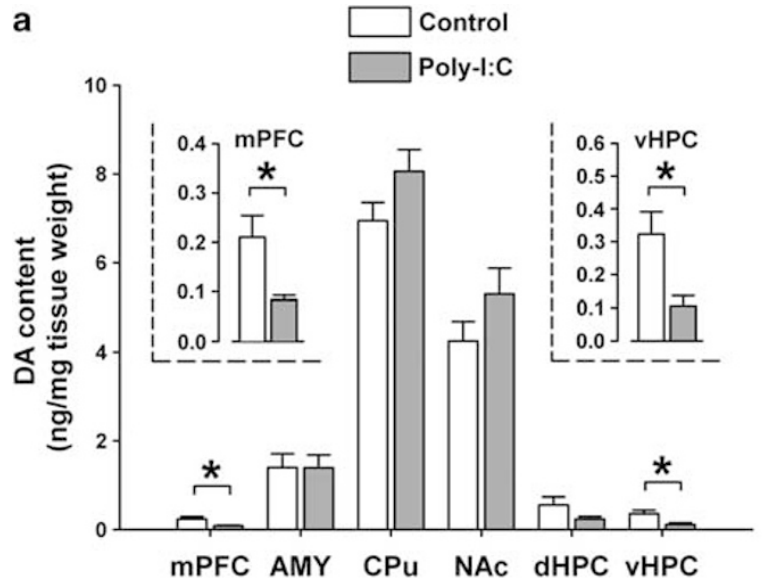

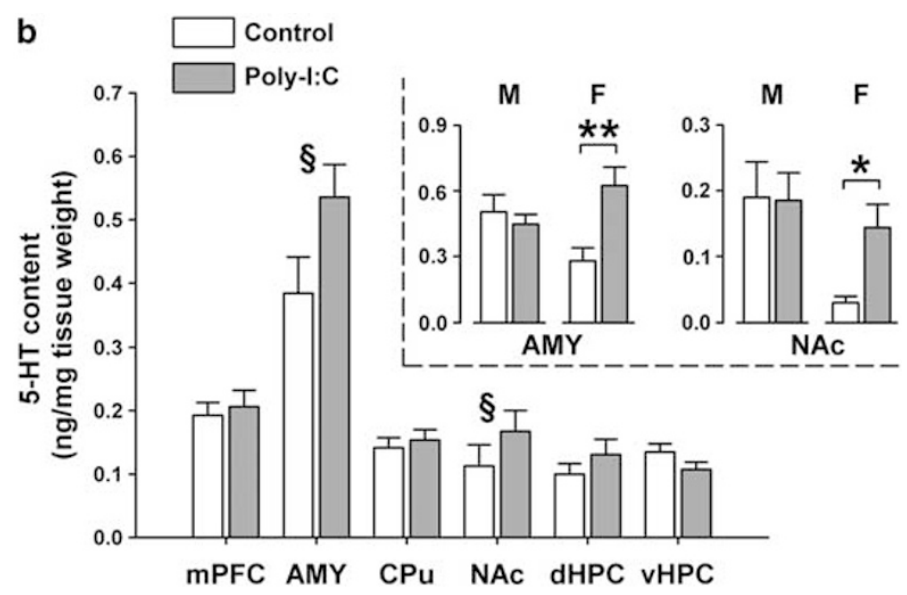

Figure 5 Effects of late prenatal immune challenge on basal dopamine (DA) and serotonin (5-HT) levels. Levels of DA and 5-HT were determined in postmortem brain tissue using high-performance liquid chromatography (HPLC). Monoamine contents were measured in the mPFC, AMY, CPu, NAc, $\mathrm{dHPC}$, and VHPC. All monoamine levels are expressed as ng per mg fresh tissue weight. (a) DA contents in adult control and Poly-l:C offspring. $* P<0.05$, based on ANOVA of DA content in the corresponding brain area. The inlets provide DA levels in the mPFC and vHPC at higher magnification. (b) 5-HT contents in adult control and Poly-l:C offspring. Symbol (\$) signifies the presence of sex-dependent effects, as further depicted by the inlets showing $5-\mathrm{HT}$ levels in the AMY and NAc of male (M) and female (F) offspring born to Poly-l:C-exposed mothers or control mothers. *P $<0.05$, based on the ANOVA restricted to females. All values are means \pm SEM.

C-treated mothers displayed a significant enhancement of 5 - $\mathrm{HT}$ in the AMY $(\mathrm{F}(5,115)=9.65, P<0.01)$ and NAc $(\mathrm{F}(1,13)=7.47, P<0.05)$ relative to levels measured in female control offspring (Figure 5b). No significant differences were found in other brain regions of Poly-I: C-exposed females compared with control females (Figure 5b). Prenatal Poly-I:C treatment did not significantly influence 5-HT levels in male offspring (Figure 5b). RM-ANOVA restricted to males failed to detect a significant main effect and/or interaction with brain area.

Monoamine metabolites and metabolites/monoamine ratios. Prenatal immune activation significantly affected the levels of 5-HIAA depending on the brain area investigated. Statistical support for this impression was obtained by the RM-ANOVA, which revealed a significant interaction between prenatal treatment and brain area $(\mathrm{F}(5,115)=2.61, P<0.05)$. Subsequent ANOVAs restricted to individual brain areas confirmed that prenatal Poly-I:C exposure significantly enhanced 5-HIAA contents specifically in the AMY (main effect of prenatal treatment: $\mathrm{F}(1,23)=4.59, P<0.05)$, but not in the other brain areas investigated. The levels of metabolites (DOPAC, HVA, 3-MT, and 5-HIAA) and monoamine/metabolite ratios (DOPAC/DA, HVA/DA, 3-MT/DA, 5-HIAA/5-HT) measured in Poly-I:C and control animals are summarized in Table 1.

\section{Region- and Sex-Specific Effects of Late Prenatal Immune Challenge on Excitatory Amino Acids}

Glutamate. Prenatal Poly-I:C exposure induced region- and sex-specific changes of glutamate levels (Figure 6a). Statistical support for this impression was obtained by RMANOVA, which revealed a significant interaction between prenatal treatment and brain area $(\mathrm{F}(5,135)=5.98$, $P<0.05)$ and between prenatal treatment, brain area, and sex $(F(5,135)=2.81, P<0.05)$. Subsequent RM-ANOVAs restricted to male and female subjects further indicated that prenatal Poly-I:C treatment led to region-specific changes in glutamate levels in both sexes, as supported by the presence of significant interactions between prenatal treatment and brain area (males: $\mathrm{F}(5,70)=2.77, P<0.05$; females: $\mathrm{F}(5,65)=3.05, P<0.05)$. Separate ANOVAs conducted for each individual brain area showed that prenatal Poly-I:C treatment significantly $(\mathrm{F}(1,14)=17.84, P<0.001)$ reduced glutamate levels in the mPFC of males but not female offspring (Figure 6a), whereas the prenatal manipulation led to a significant decrease in glutamate levels in the dHPC of both male and female subjects (males: $\mathrm{F}(1,14)=10.71$, $P<0.01$; females: $\mathrm{F}(1,13)=8.81, P<0.01$; Figure 6a). No other significant effects were observed in the analyses of glutamate.

Aspartate. Prenatal Poly-I:C exposure also induced regionand sex-specific changes in aspartate levels (Figure 6b). Statistical support for this interpretation was obtained by RM-ANOVA, which yielded a significant interaction between prenatal treatment and brain area $(\mathrm{F}(5,140)=3.48$, $P<0.01)$ and between prenatal treatment, brain area, and sex $(\mathrm{F}(5,140)=2.72, P<0.05)$. Subsequent RM-ANOVAs restricted to male and female subjects further indicated that prenatal Poly-I:C treatment led to region-specific changes in aspartate levels in both sexes, as supported by the presence of significant interactions between prenatal treatment and brain area (males: $\mathrm{F}(5,70)=3.27, P<0.05$; females: $\mathrm{F}(5,70)=2.925, P<0.05)$. Separate ANOVAs conducted for individual brain areas verified that prenatal Poly-I:C exposure significantly $(F(1,14)=17.84, P<0.001)$ reduced aspartate levels in the mPFC of males but not female offspring (Figure $6 \mathrm{~b}$ ), whereas the prenatal manipulation led to a significant decrease in aspartate levels in the dHPC of both male and female subjects (males: $\mathrm{F}(1,14)=7.56$, $P<0.01$; females: $\mathrm{F}(1,14)=9.31, P<0.01$; Figure $6 \mathrm{~b})$. No other significant effects were observed in the analyses of aspartate. 
Table I Mean \pm SEM Values (in ng/mg tissue) of Monoamine Metabolites and Monoamine/Metabolite Ratios in the Distinct Brain Regions of Adult Control Offspring and Offspring Subjected to Prenatal Poly-l:C Treatment in Late Gestation

\begin{tabular}{|c|c|c|c|c|c|c|c|c|}
\hline & DOPAC & HVA & 3-MT & DOPAC/DA & HVA/DA & 3-MT/DA & 5-HIAA & 5-HIAA/5HT \\
\hline \multicolumn{9}{|l|}{ mPFC } \\
\hline \multicolumn{9}{|l|}{ Control } \\
\hline Female & $0.19 \pm 0.06$ & $0.15 \pm 0.03$ & $0.03 \pm 0.01$ & $1.06 \pm 0.28$ & $0.92 \pm 0.21$ & $0.17 \pm 0.04$ & $0.14 \pm 0.02$ & $0.94 \pm 0.10$ \\
\hline \multicolumn{9}{|l|}{ Polyl:C } \\
\hline \multicolumn{9}{|l|}{ AMY } \\
\hline \multicolumn{9}{|l|}{ Control } \\
\hline Male & $0.30 \pm 0.07$ & $0.31 \pm 0.05$ & $0.09 \pm 0.02$ & $0.50 \pm 0.09$ & $0.40 \pm 0.06$ & $0.11 \pm 0.02$ & $0.23 \pm 0.04$ & $0.55 \pm 0.08$ \\
\hline Female & $0.80 \pm 0.24$ & $0.48 \pm 0.10$ & $0.17 \pm 0.04$ & $0.52 \pm 0.08$ & $0.32 \pm 0.04$ & $0.11 \pm 0.02$ & $0.24 \pm 0.04$ & $0.92 \pm 0.06$ \\
\hline
\end{tabular}

$\mathrm{CPu}$

Control

Male

Female

$$
0.96 \pm 0.12
$$

$0.70 \pm 0.08$

$0.25 \pm 0.02$

$0.13 \pm 0.01$

$0.10 \pm 0.01$

$0.04 \pm 0.00$

$0.13 \pm 0.02$

$0.77 \pm 0.03$

Polyl:C

Male

$1.05 \pm 0.05$

$0.79 \pm 0.07$

$0.30 \pm 0.02$

$0.13 \pm 0.01$

$0.12 \pm 0.01$

$0.05 \pm 0.00$

$0.09 \pm 0.01$

$0.78 \pm 0.04$

Female

$2.21 \pm 0.43$

$0.90 \pm 0.08$

$0.32 \pm 0.02$

$0.09 \pm 0.01$

$0.04 \pm 0.00$

$0.12 \pm 0.02$

$0.7 \mid \pm 0.03$

$0.04 \pm 0.01$

$0.12 \pm 0.02$

$0.87 \pm 0.06$

$N A c$

Control

\section{Male}

Female

$1.07 \pm 0.14$

$0.72 \pm 0.07$

$0.22 \pm 0.02$

$0.21 \pm 0.03$

$0.13 \pm 0.01$

$0.04 \pm 0.00$

$0.13 \pm 0.02$

$0.72 \pm 0.13$

$0.19 \pm 0.05$

$0.61 \pm 0.13$

$0.21 \pm 0.03$

$0.06 \pm 0.01$

$0.05 \pm 0.02$

$2.39 \pm 0.81$

\section{Male}

$1.02 \pm 0.16$

$0.75 \pm 0.11$

$0.22 \pm 0.03$

$0.17 \pm 0.02$

$0.12 \pm 0.01$

$0.04 \pm 0.00$

$0.08 \pm 0.03$

$0.41 \pm 0.06$

Female

$2.10 \pm 0.57$

$0.81 \pm 0.19$

$0.25 \pm 0.06$

$0.17 \pm 0.02$

$0.17 \pm 0.02$

$0.05 \pm 0.01$

$0.10 \pm 0.04$

$0.79 \pm 0.15$

IHPC

Control

Male

Female

$0.27 \pm 0.06$

$0.16 \pm 0.03$

$0.07 \pm 0.02$

$0.75 \pm 0.25$

$0.45 \pm 0.14$

$0.16 \pm 0.06$

$0.12 \pm 0.01$

$0.98 \pm 0.12$

Polyl:C

Male

$0.17 \pm 0.04$

$0.12 \pm 0.03$

$0.06 \pm 0.01$

$0.76 \pm 0.11$

$0.50 \pm 0.09$

$0.36 \pm 0.07$

$0.10 \pm 0.02$

$1.44 \pm 0.15$

Female

$0.14 \pm 0.01$

$0.10 \pm 0.01$

$0.04 \pm 0.00$

$1.22 \pm 0.23$

$0.21 \pm 0.05$

$0.13 \pm 0.02$

$0.96 \pm 0.06$

$0.88 \pm 0.18$

$0.41 \pm 0.10$

$0.14 \pm 0.02$

$1.38 \pm 0.18$

$\checkmark H P C$

Control

\begin{tabular}{ccccccccc} 
Male & $0.16 \pm 0.03$ & $0.11 \pm 0.02$ & $0.06 \pm 0.01$ & $0.41 \pm 0.05$ & $0.29 \pm 0.05$ & $0.15 \pm 0.02$ & $0.13 \pm 0.01$ & $0.83 \pm 0.06$ \\
Female & $0.17 \pm 0.05$ & $0.10 \pm 0.02$ & $0.06 \pm 0.01$ & $1.05 \pm 0.16$ & $0.81 \pm 0.21$ & $0.34 \pm 0.08$ & $0.12 \pm 0.02$ & $1.14 \pm 0.09$ \\
Polyl:C & & & & & & & 0.02 & \\
Male & $0.08 \pm 0.02$ & $0.06 \pm 0.01$ & $0.03 \pm 0.00$ & $0.78 \pm 0.16$ & $0.80 \pm 0.16$ & $0.35 \pm 0.08$ & $0.09 \pm 0.01$ & $1.02 \pm 0.07$ \\
Female & $0.10 \pm 0.02$ & $0.08 \pm 0.01$ & $0.04 \pm 0.00$ & $1.63 \pm 0.29$ & $1.45 \pm 0.31$ & $0.59 \pm 0.17$ & $0.15 \pm 0.02$ & $1.20 \pm 0.09$ \\
\hline
\end{tabular}

Abbreviations: AMY, amygdala; CPu, caudate putamen; DA, dopamine; dHPC, dorsal hippocampus; DOPAC, dihydroxyphenylacetic acid; HVA, homovanillic acid; mPFC, medial prefrontal cortex; NAc, nucleus accumbens; vHPC, ventral hippocampus; 5-HIAA, 5-hydroxyindoleacetic acid; 5-HT, 5-hydroxytryptamine; 3-MT, 3-methoxytyramine.

Prenatal Poly-l:C exposure significantly enhanced 5-HIAA contents specifically in the AMY but not in the other brain areas investigated.

*** $P<0.0$ I, based on the ANOVA of 5-HIAA levels in the AMY of male and female offspring. 

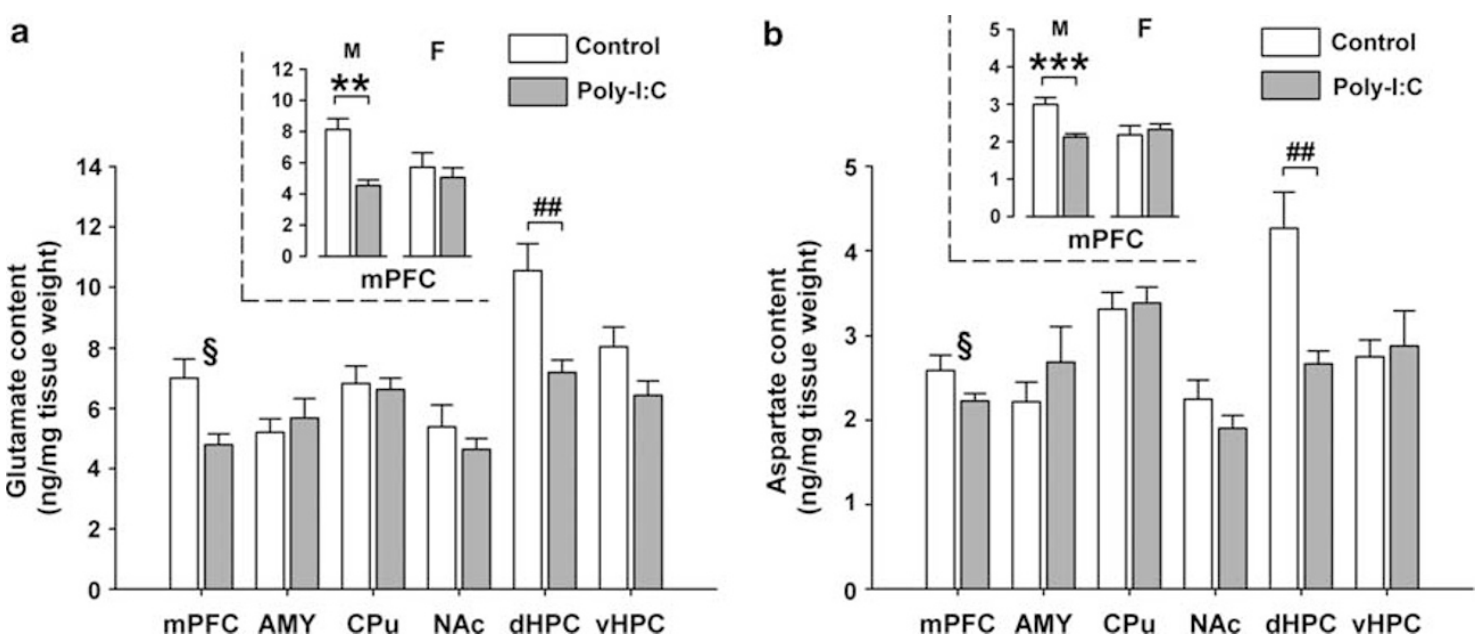

Figure 6 Effects of late prenatal immune challenge on basal levels of excitatory amino acids. Levels of the excitatory amino acids glutamate and aspartate were determined in postmortem brain tissue using HPLC. The contents of these excitatory amino acids were measured in the mPFC, AMY, CPu, NAc, $\mathrm{dHPC}$, and vHPC. All levels are expressed as ng per mg fresh tissue weight. (a) Glutamate contents in adult control and Poly-l:C offspring. Symbol (\$) signifies the presence of a sex-dependent effect in the mPFC, as further depicted by the inlets showing glutamate levels in the mPFC of male (M) and female (F) offspring born to Poly-l:C-exposed mothers or control mothers. $* * P<0.01$, based on the ANOVA restricted to males. ${ }^{\#} P<0.01$, based on ANOVA in males and females. (b) Aspartate contents in adult control and Poly-l:C offspring. Symbol (§) signifies the presence of a sex-dependent effect in the mPFC, as further depicted by the inlets showing aspartate levels in the mPFC of male (M) and female (F) offspring born to Poly-l:C-exposed mothers or control mothers. *** $P<0.001$, based on the ANOVA restricted to males. ${ }^{\#} P<0.01$, based on ANOVA of dHPC contents in males and females. All values are means $\pm \mathrm{SEM}$.

\section{Region- and Sex-Specific Effects of Late Prenatal Immune Challenge on Inhibitory Amino Acids}

Glycine. Prenatal Poly-I:C exposure did not significantly affect the brain levels of glycine (data not shown). RMANOVA of glycine contents did not reveal a significant main effect or interactions involving the between-subjects factor of prenatal treatment.

$G A B A$. Prenatal immune activation in late gestation exerted region- and sex-specific effects on GABA levels, as supported by the presence of a significant interaction between prenatal treatment, sex, and brain region $(\mathrm{F}(5,140)=3.42$, $P<0.01)$ in the RM-ANOVA of GABA contents. By revealing a significant interaction between prenatal treatment and brain areas $(\mathrm{F}(5,70)=3.52, P<0.05)$, the subsequent RMANOVA restricted to female subjects further indicated that prenatal Poly-I:C treatment led to region-specific changes in GABA levels in female offspring. Separate ANOVAs conducted for individual brain areas in females showed that female offspring prenatally exposed to Poly-I:C treatment displayed a significant $(\mathrm{F}(1,14)=7.81, P<0.05)$ reduction in GABA levels specifically in the dHPC relative to female control offspring (Figure 7a). On the other hand, the lack of any statistically significant effects in the RMANOVA restricted to male subjects suggested that prenatal immune activation did not significantly affect GABA levels in males (Figure 7a).

Taurine. Prenatal Poly-I:C treatment also induced regionand sex-specific effects on brain taurine contents. Statistical support for this impression was obtained by RM-ANOVA of taurine levels, which revealed a significant interaction between prenatal treatment and brain region $(\mathrm{F}(5,140)=$ 2.91, $P<0.05)$ and between prenatal treatment, sex, and brain region $(F(5,140)=3.51, P<0.01)$. Subsequent
RM-ANOVAs restricted to male and female subjects further indicated that prenatal Poly-I:C treatment led to regionspecific changes in taurine levels in both sexes, as supported by the presence of significant interactions between prenatal treatment and brain area (males: $\mathrm{F}(5,70)=3.69, P<0.01$; females: $F(5,70)=2.51, P<0.05)$. Separate ANOVAs conducted for individual brain areas verified that prenatal PolyI:C exposure significantly $(F(1,14)=10.04, P<0.01)$ reduced taurine levels in the mPFC of males (Figure $7 \mathrm{~b}$ ), whereas the prenatal manipulation led to a significant decrease in taurine levels in the dHPC of female subjects $(\mathrm{F}(1,14)=$ $5.83, P<0.05$; Figure $7 \mathrm{~b}$ ). No other significant effects were observed in the analyses of taurine.

\section{DISCUSSION}

The present experimental investigation in mice demonstrates that exposure to a viral-like acute phase response by Poly-I:C treatment in late gestation leads to long-term deficits in social interaction, anhedonic behavior in the sucrose preference test, presence of abnormally enhanced LI in associative learning, and alterations in the locomotor and stereotyped behavioral responses to acute APO treatment. These effects extend our previous observations that late prenatal Poly-I:C-induced immune challenge in mice is capable of inducing cognitive abnormalities in the form of deficient spatial working and recognition memory (Bitanihirwe et al, 2010; Meyer et al, 2008c, 2010), impaired discrimination reversal learning (Meyer et al, 2006b), and enhanced behavioral sensitivity to acute treatment with the indirect DA receptor agonist amphetamine and the NMDAreceptor antagonist MK-801 (Meyer et al, 2008c). The present study thus identified several novel phenotypes which are part of a pathological symptom cluster characteristic for prenatal Poly-I:C-induced immune activation 

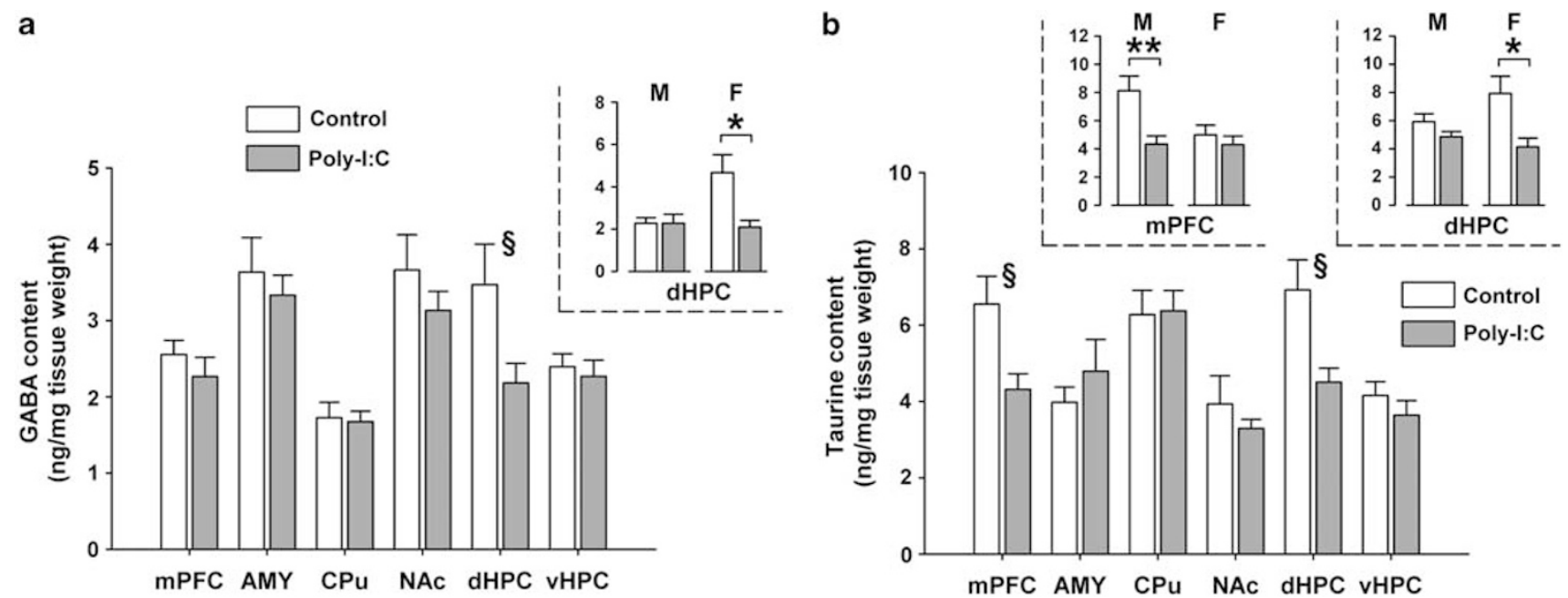

Figure 7 Effects of late prenatal immune challenge on basal levels of inhibitory amino acids. Levels of the inhibitory amino acids GABA and taurine were determined in postmortem brain tissue using HPLC. The contents of these inhibitory amino acids were measured in the mPFC, AMY, CPu, NAc, dHPC, and VHPC. All levels are expressed as ng per mg fresh tissue weight. (a) GABA contents in adult control and Poly-l:C offspring. Symbol (§) signifies the presence of a sex-dependent effect in the dHPC, as further depicted by the inlets showing GABA levels in the dHPC of male (M) and female (F) offspring born to Poly-l:C-exposed mothers or control mothers. $* P<0.05$, based on the ANOVA restricted to females. (b) Taurine contents in adult control and Poly-l:C offspring. Symbol (\$) signifies the presence of sex-dependent effects, as further depicted by the inlets showing GABA levels in the mPFC and dHPC of male $(\mathrm{M})$ and female (F) offspring born to Poly-l:C-exposed mothers or control mothers. ${ }^{*} * P<0.0 \mathrm{I}$, based on the ANOVA restricted to males; ${ }^{*} P<0.0 \mathrm{I}$, based on the ANOVA restricted to females. All values are means \pm SEM.

in late gestation. Our detailed postmortem neurochemical analyses further show that the behavioral and pharmacological abnormalities emerging after late prenatal immune challenge are associated with various changes in basal neurotransmitter levels. This confirms the expectation that the appearance of multiple behavioral and pharmacological abnormalities following prenatal immune challenge is paralleled by multiple neurochemical disturbances.

One of the main findings of the present study is that adult offspring of mothers treated with Poly-I:C in late gestation (ie GD17) display LI under parametric conditions which are insufficient to induce the LI effect in control offspring. Hence, late prenatal Poly-I:C exposure in mice leads to abnormally enhanced LI under such conditions. According to the 'switching model' of LI proposed by Weiner (1990, 2003), the development of LI involves the acquisition of two independent and conflicting contingencies in pre-exposure and conditioning, which then compete for expression during conditioning. To show LI, the organism must remain under the control of the information acquired in pre-exposure. Since during the pre-exposure phase the CS is not followed by a significant event, the information acquired in pre-exposure relates to a stimulus-no event contingency. In contrast, absence of LI would indicate that the organism switches to respond according to the new stimulus-event (ie, CS-US) contingency present during conditioning. According to these theoretical accounts, manipulations that delay switching and thus induce behavioral/cognitive perseveration facilitate the expression of LI under parametric conditions in which control subjects fail to show LI. It follows that the abnormally enhanced LI effect manifested in prenatally Poly-I:C-treated offspring may readily be accounted for by such perseveration effects, so that CS-pre-exposed Poly-I:C subjects remain under the control of information acquired in pre-exposure (stimulus-no event) and therefore display impaired learning during the critical CS-US conditioning phase. The comparison between conditioned learning in CS-pre-exposed PolyI:C and control offspring further supports this possibility and suggests that the presence of LI in Poly-I:C offspring exclusively stems from a specific effect of the prenatal manipulation on conditioned learning in CS-PE subjects (Figure 3). Furthermore, the current interpretation of perseveration effects in LI are in agreement with our previous findings showing that prenatal Poly-I:C-induced immune activation in late gestation leads to perseverative behavior in the form of delayed reversal learning (Meyer et al, 2006b).

LI is known to be a window phenomenon, in which the stimulus-no event association acquired in pre-exposure is only expressed under a very specific balance between the behavioral impact of pre-exposure and conditioning (Weiner, 1990, 2003). Thus, LI is expressed only with specific combinations of pre-exposure and conditioning parameters (conditions denoted by the switching model as 'low mismatch', which for example can occur as a result of extensive stimulus pre-exposure or minimal conditioning and/or low impact of reinforcement); changes in any of these parameters such as restricting the amount of stimulus pre-exposure or extending conditioning (conditions denoted by the switching model as 'high mismatch') can cause the organism to switch responding according to the stimulus-reinforcement contingency and to cease to express LI. In one of our previous attempts to characterize the longterm brain and behavioral consequences of prenatal PolyI:C exposure in mice, we used a LI paradigm with extensive CS-PE which was effective to produce a robust LI effect in adult control offspring (Meyer et al, 2006a). Under such parametric conditions, prenatal Poly-I:C treatment on GD17 did not significantly enhance LI compared with prenatal 
control treatment (Meyer et al, 2006a). It is important to emphasize that this 'lack of effect' in our earlier study is not surprising, nor is it inconsistent with the present data. Indeed, parametric conditions which induce a robust LI effect in control subjects often impede the identification of potential LI enhancement/persistence in manipulated subjects because of possible ceiling effects (Weiner, 2003). In the present study, we therefore used parametric conditions with low amount of CS-PE to facilitate the identification of potential LI enhancement/persistence in offspring subjected to prenatal Poly-I:C exposure in late gestation.

Several studies in humans have shown that LI is disrupted in acutely ill schizophrenic patients with marked positive symptoms (reviewed in Weiner, 2003; Lubow, 2005). Hence, under parametric conditions in which healthy human subjects show a significant LI effect, acutely psychotic patients fail to do so (reviewed in Weiner, 2003; Lubow, 2005). This resembles the long-term consequences of prenatal Poly-I:C treatment in early/middle gestation in mice and rats, which lead to a loss of the LI effect under parametric conditions in which adult control subjects display significant LI (for mice see eg, Meyer et al, 2005, 2006a; Smith et al, 2007; for rats, see Zuckerman et al, 2003; Zuckerman and Weiner, 2005). On the other hand, the presence of abnormally enhanced LI positively correlates with the severity of negative symptoms in (chronically ill) patients with schizophrenia (Cohen et al, 2004; Rascle et al, 2001). Based on these findings in humans and parallel experimental investigations in rats and mice (GaislerSalomon and Weiner, 2003; Gaisler-Salomon et al, 2008; Lipina et al, 2005), it has been suggested that the presence of abnormally enhanced LI may provide a correlate of a specific aspect of negative symptomatology, namely, behavioral or attentional perseveration (Weiner, 2003; Weiner and Arad, 2009). Against this background, the presence of abnormally enhanced LI in adult offspring subjected to prenatal Poly-I:C treatment in late gestation suggests that this prenatal immunological manipulation can induce specific behavioral/cognitive abnormalities directly implicated in the negative symptoms of schizophrenia. Interestingly, this LI perseveration effect only emerged in male but not female offspring born to Poly-I:C-treated mothers, indicating that the male sex is more vulnerable than the female sex to develop such behavioral/cognitive abnormalities. This sex-dependent effect of prenatal Poly$\mathrm{I}: \mathrm{C}$ treatment is consistent with the clinical impressions that male schizophrenic patients seem to exhibit a more severe clinical profile compared with female patients (Aleman et al, 2003; Flor-Henry, 1990), especially in terms of negative symptomatology and cognitive deficits (Roy et al, 2001). However, the efficacy of prenatal Poly-I:C treatment to induce sex-specific abnormalities appears to be specific to the behavioral and/or cognitive processes involved in LI, as maternal Poly-I:C exposure induced social interaction deficits and anhedonic behavior similarly in male and female offspring.

Using a social interaction test which is based on the relative exploration of an unfamiliar live mouse $v s$ an inanimate dummy object, we found that whereas control offspring spent significantly more time exploring the live mouse, prenatally Poly-I:C-treated offspring displayed no preference towards either objects (Figure 1). Hence, offspring born to Poly-I:C-exposed mothers spent equal amounts of time exploring the live mouse and the inanimate dummy object, indicating a marked disruption of normal social behavior. This effect is consistent with previous reports of social interaction deficits emerging in adult mice exposed to Poly-I:C in middle gestation (GD 12; Smith et al, 2007) or to the bacterial endotoxin lipopolysaccharide in late gestation (GD 17; Hava et al, 2006). Notably, deficient social interaction is one of the hallmark negative symptoms in schizophrenia (Foussias and Remington, 2010; McGlashan and Fenton, 1992; Schooler and Spohn, 1982). Therefore, the presence of genuine social interaction deficits revealed in Poly-I:C offspring provides further direct support for our proposal that late prenatal immune challenge in mice can induce long-term behavioral abnormalities relevant to the negative symptoms of this disorder.

To the best of our knowledge, the present study is the first to demonstrate significant long-term effects of prenatal immune activation in the sucrose preference test of anhedonia. This test has been shown to be a sensitive experimental paradigm to evaluate hedonic aspects of feeding behavior in mice (Cryan and Mombereau, 2004; Hayward et al, 2006; Pothion et al, 2004). Indeed, when presented with a free choice between sucrose solution and water, mice (and other rodent species) typically show a marked preference for the sucrose solution. Consistent with this natural tendency of rodents, we found that both PolyI:C and control offspring preferred the sucrose solution over the water solution during the choice test phase: the preference index for sucrose consumption was clearly above a level of $50 \%$ indifference in both prenatal treatment groups (Figure 2). Most importantly, however, prenatally immune challenged offspring showed a significant $(\sim 15 \%)$ reduction in the sucrose preference index relative to control offspring. As a reduction in this preference index is often indicative of anhedonic behavior (Cryan and Mombereau, 2004; Hayward et al, 2006; Pothion et al, 2004), our findings suggest that late prenatal exposure to a viral-like acute phase response may facilitate the expression of anhedonia in adulthood. Anhedonia has often been discussed to be another hallmark feature of negative symptoms in schizophrenia (Foussias and Remington, 2010; Horan et al, 2008; Juckel et al, 2003; McGlashan and Fenton, 1992). Hence, late prenatal Poly-I:C treatment in mice also successfully captures this specific aspect of behavioral pathology seen in schizophrenic patients with marked negative symptoms.

Another previously unexplored pathological phenotype emerging after late prenatal Poly-I:C treatment in mice is alterations in the locomotor and stereotyped behavioral reactions to acute APO treatment (Figure 4). Consistent with previous reports in C57BL/6 mice (Cabib and PuglisiAllegra, 1985; Protais et al, 1976), we found that acute APO treatment at the selected dose $(5 \mathrm{mg} / \mathrm{kg}$, s.c.) led to a pronounced increase in repetitive climbing (Figure $4 \mathrm{~b}$ ). Most importantly, we revealed that adult Poly-I:C offspring displayed a faster onset of APO-induced climbing compared with APO-treated control offspring (Figure $4 \mathrm{~b}$ ). This effect was paralleled by a temporary attenuation of leaning behavior in APO-induced Poly-I:C offspring relative to APO-treated controls (Figure 4c). Poly-I:C and control offspring also differed in terms of the horizontal locomotor response to acute APO treatment, with APO-treated 
Poly-I:C offspring showing an overall increase in horizontal movements relative to APO-treated control offspring (Figure 4a). Taken together, our findings suggest that prenatal Poly-I:C treatment in late gestation is effective in enhancing the sensitivity to acute APO treatment both in terms of stereotyped behavioral responses as well as horizontal locomotor activity. In relation to the clinical pathology in humans, it is interesting to note that several (but not all) studies have reported enhanced sensitivity of schizophrenic patients to systemic APO treatment, as assessed by APO-stimulated peripheral growth hormone (GH) responses (Hirschowitz et al, 1986; Meltzer et al, 1984; Zemlan et al, 1986). Interestingly, APO-induced GH responses appear to positively correlate with psychosis ratings and negative symptom scale scores (Meltzer et al, 1984). This clinical impression agrees well with the present interpretation that (1) experimentally induced prenatal immune challenge in mice leads to long-term behavioral and pharmacological changes relevant to the negative symptoms of schizophrenia and (2) this experimentally induced pathological symptom cluster includes enhanced sensitivity to acute APO treatment.

Another major finding of the present study is that late prenatal immune activation leads to long-term neurochemical changes in multiple neurotransmitter systems (Figures 5-7). However, the prenatal Poly-I:C-induced changes in neurotransmitter levels were dependent on the precise brain area examined, suggesting that distinct brain structures may have a differential vulnerability for prenatal infectioninduced imbalances in basal neurotransmitter levels. This interpretation is consistent with previous experimental attempts to characterize the long-term neurochemical consequences of prenatal immune challenge during early fetal development in mice (Winter et al, 2009) and of prenatal exposure to human influenza in mice (Fatemi et al, 2008; Winter et al, 2008). Furthermore, we found that some of the Poly-I:C-induced neurochemical changes are clearly sex-specific, highlighting a differential vulnerability of the male and female sex to long-term neurochemical abnormalities emerging after late prenatal immune challenge.

Besides other significant changes, the neurochemical abnormalities present in prenatally immune challenged offspring are characteristic of marked hypodopaminergic (Figure 5b) and hypoglutamatergic (Figure 6a) states in prefrontal and hippocampal regions. Converging evidence derived from studies in humans and translational animal models suggests that such hypodopaminergic and hypoglutamatergic states in prefrontal and hippocampal areas may be critically involved especially in the precipitation of negative and cognitive symptoms of schizophrenia (reviewed in Davis et al, 1991; Goff and Coyle, 2001; Knable and Weinberger, 1997; Tamminga, 2006). Hence, the results obtained in the analyses of DA and glutamate provide imperative evidence that the long-term abnormalities induced by late prenatal immune challenge in mice involve neurochemical changes highly relevant for the pathophysiology of negative symptoms.

We deem it likely that the identified neurotransmitter changes in Poly-I:C offspring may, at least in part, provide a neurochemical basis for the emergence of behavioral pathology described here. For example, reduced glutamatergic signaling may be critically involved in the emergence of abnormally enhanced LI. This suggestion would be consistent with the seminal work by Weiner and colleagues showing that impaired glutamatergic signaling induced by MK-801 treatment leads to a similar pattern of LI enhancement in rats and mice (Gaisler-Salomon and Weiner, 2003; Gaisler-Salomon et al, 2008; Lipina et al, 2005). Interestingly, the effects of prenatal immune challenge on glutamatergic changes appear more pronounced in males compared with female subjects (Figure 6a), and these effects parallel the sex-dependent effects of prenatal immune challenge on LI persistence (Figure 3). On the other hand, a reduced dopaminergic tone may be involved in the expression of anhedonia, based on the suggested role of DA in rewardrelated behavior (Berridge and Robinson, 1998; Cagniard et al, 2006; Wise, 2008), as well as in the development of social interaction deficits (Cabib et al, 2000; Fernandez Espejo, 2003). Additional pharmacological and/or in-vivo microdialysis approaches will be needed to further dissect the relative contribution of distinct neurochemical abnormalities to specific behavioral and pharmacological abnormalities in prenatally immune challenged subjects.

One limitation of the present study is that we did not include different postnatal ages in our phenotypic characterization of the effects of late prenatal immune challenge. Hence, the ontogeny of the various behavioral, pharmacological, and neurochemical abnormalities identified in adult offspring subjected to late prenatal Poly-I:C treatment remain essentially unknown thus far. Given that a hallmark feature of schizophrenia is the post-pubertal maturational delay of symptom onset, it will be highly relevant to extend the present study by including phenotypic characterizations at different pre- and post-pubertal stages of development.

As discussed in detail elsewhere (Meyer et al, 2007, 2009a, b; Meyer and Feldon, 2009, 2010), there are several plausible mechanisms whereby prenatal Poly-I:C-induced immune activation can bring about changes in brain and behavioral development relevant to schizophrenia. Above all, converging evidence derived from several lines of research emphasizes a critical role of enhanced expression of pro-inflammatory cytokines in the precipitation of longterm brain dysfunctions following prenatal Poly-I:Cinduced immune challenge (Meyer et al, 2006b, 2008a; Shi et al, 2003; Smith et al, 2007). According to the prenatal cytokine imbalance hypothesis (Meyer et al, 2009b), a shift towards excess levels of pro-inflammatory cytokines during fetal life can interfere with fetal neurodevelopmental processes and predispose the organisms to long-term changes in subsequent brain and behavioral development. Recent longitudinal investigations provide direct support for this hypothesis by showing that maternal Poly-I:Cinduced immune challenge is capable of directly affecting the development of fetal brain regions and neurotransmitter systems, which are involved in the precipitation of schizophrenia-related behavioral, cognitive, and pharmacological abnormalities at adult age (Vuillermot et al, 2010). Nevertheless, even though evidence for a critical role of pro-inflammatory cytokines in this context is compelling, alternative (but not mutually exclusive) mechanisms exist whereby prenatal exposure to (Poly-I:C-induced) prenatal immune challenge may affect brain and behavioral development. Such alternative mechanisms include infectioninduced stimulation of maternal/fetal stress response 
systems as well as (short-term) placental insufficiency and maternal/fetal nutritional deprivation, all of which are also known to exert an impact on early brain development (Brown and Susser, 2008; Koenig et al, 2002; Patterson, 2007; Susser et al, 2008). Therefore, additional cellular and molecular studies will be needed to further dissect the relative contribution of specific neuroimmunological, endocrinological, and developmental factors to the precipitation of schizophrenia-related brain dysfunctions induced by prenatal immune challenge.

In summary, the constellation of behavioral and neurochemical abnormalities induced by late prenatal Poly-I:C exposure in mice reported here and previously (Meyer et al, 2006a, b, 2008b) leads us to conclude that this immunebased experimental model provides a powerful neurodevelopmental animal model especially for (but not limited to) the negative symptoms of schizophrenia. This model can successfully capture a wide spectrum of behavioral and neurochemical abnormalities implicated in the negative symptoms of this disorder. Importantly, it incorporates neurodevelopmental perspectives and etiological significance. The late prenatal Poly-I:C model in mice thus represents a clear improvement over the limited success and etiological relevance of traditional pharmacological or lesion-based experimental approaches in modeling brain and behavioral abnormalities relevant to the negative symptoms of schizophrenia (Ellenbroek and Cools, 2000). As current pharmacotherapy of negative symptoms remains unsatisfactory (Buchanan et al, 2007; Remington et al, 2010), the late prenatal Poly-I:C model in mice may provide fruitful new avenues for the establishment and evaluation of novel antipsychotic compounds in attempts to improve pharmacological treatments for the negative symptoms of schizophrenia. It also interesting to note that our experimental studies reported here and previously (Meyer et al, 2006a, b, 2008b) suggest that prenatal viral-like immune challenge taking place during early fetal development may particularly enhance the vulnerability and/or severity of positive symptoms in schizophrenia, whereas identical immune challenge during later periods of fetal development may be more critical for shaping the vulnerability for negative/cognitive symptoms of this disorder. In support for the latter suggestion, there is preliminary evidence that the levels of negative symptoms such as anhedonia are exaggerated in schizophrenic patients who were born in winters with high infectious disease rates (Watson et al, 1987), pointing to infection-related effects taking place in late gestation. Our experimental data may thus also be relevant for future epidemiological attempts to dissect the relative contribution of prenatal infectious processes to distinct symptom clusters and/or brain pathologies in schizophrenia and related disorders.

\section{ACKNOWLEDGEMENTS}

This study was supported by the Swiss National Science Foundation (Grant 3100AO-100309 and Grant 3100A0116719), by the Swiss Federal Institute of Technology (Grant $1107 / 03$ ) and by a 2009 NARSAD Distinguished Investigator Award to Joram Feldon. We are extremely grateful to Stéphanie Vuillermot and Celia Hampson-Reid for technical support. We are also indebted to the Animal Services Department Schwerzenbach, Swiss Federal Institute of Technology, for their excellent animal husbandry and care.

\section{DISCLOSURE}

The authors declare no conflict of interest.

\section{REFERENCES}

Aleman A, Kahn RS, Selten JP (2003). Sex differences in the risk of schizophrenia: evidence from meta-analysis. Arch Gen Psychiatry 60: 565-571.

Arguello PA, Gogos JA (2006). Modeling madness in mice: one piece at a time. Neuron 52: 179-196.

Berridge KC, Robinson TE (1998). What is the role of dopamine in reward: hedonic impact, reward learning, or incentive salience? Brain Res Brain Res Rev 28: 309-369.

Bitanihirwe BK, Weber L, Feldon J, Meyer U (2010). Cognitive impairment following prenatal immune challenge in mice correlates with prefrontal cortical AKT1 deficiency. Int J Neuropsychopharmacol in press (Epub ahead of print [PMID: 20219156]).

Blanchard JJ, Cohen AS (2006). The structure of negative symptoms within schizophrenia: implications for assessment. Schizophr Bull 32: 238-245.

Brown AS (2006). Prenatal infection as a risk factor for schizophrenia. Schizophr Bull 32: 200-202.

Brown AS, Derkits EJ (2010). Prenatal infection and schizophrenia: a review of epidemiologic and translational studies. Am J Psychiatry 167: 261-280.

Brown AS, Susser ES (2008). Prenatal nutritional deficiency and risk of adult schizophrenia. Schizophr Bull 34: 1054-1063.

Buchanan RW, Javitt DC, Marder SR, Schooler NR, Gold JM, McMahon RP et al (2007). The Cognitive and Negative Symptoms in Schizophrenia Trial (CONSIST): the efficacy of glutamatergic agents for negative symptoms and cognitive impairments. Am J Psychiatry 164: 1593-1602.

Cabib S, D'Amato FR, Puglisi-Allegra S, Maestripieri D (2000). Behavioral and mesocorticolimbic dopamine responses to non aggressive social interactions depend on previous social experiences and on the opponent's sex. Behav Brain Res 112: $13-22$.

Cabib S, Puglisi-Allegra S (1985). Different effects of apomorphine on climbing behavior and locomotor activity in three strains of mice. Pharmacol Biochem Behav 23: 555-557.

Cagniard B, Balsam PD, Brunner D, Zhuang X (2006). Mice with chronically elevated dopamine exhibit enhanced motivation, but not learning, for a food reward. Neuropsychopharmacology 31: $1362-1370$.

Clancy B, Darlington RB, Finlay BL (2001). Translating developmental time across mammalian species. Neuroscience 105: 7-17.

Clancy B, Finlay BL, Darlington RB, Anand KJ (2007). Extrapolating brain development from experimental species to humans. Neurotoxicology 28: 931-937.

Cohen E, Sereni N, Kaplan O, Weizman A, Kikinzon L, Weiner I et al (2004). The relation between latent inhibition and symptom-types in young schizophrenics. Behav Brain Res 149: 113-122.

Crawley JN (2007). Mouse behavioral assays relevant to the symptoms of autism. Brain Pathol 17: 448-459.

Crider A (1997). Perseveration in schizophrenia. Schizophr Bull 23: 63-74.

Crow TJ (1980). Positive and negative schizophrenic symptoms and the role of dopamine. Br J Psychiatry 137: 383-386. 
Cryan JF, Mombereau C (2004). In search of a depressed mouse: utility of models for studying depression-related behavior in genetically modified mice. Mol Psychiatry 9: 326-357.

Dalley JW, Cardinal RN, Robbins TW (2004). Prefrontal executive and cognitive functions in rodents: neural and neurochemical substrates. Neurosci Biobehav Rev 28: 771-784.

Davis KL, Kahn RS, Ko G, Davidson M (1991). Dopamine in schizophrenia: a review and reconceptualization. Am J Psychiatry 148: 1474-1486.

Ellenbroek BA, Cools AR (2000). Animal models for the negative symptoms of schizophrenia. Behav Pharmacol 11: 223-233.

Errico F, Nisticò R, Palma G, Federici M, Affuso A, Brilli E et al (2008). Increased levels of D-aspartate in the hippocampus enhance LTP but do not facilitate cognitive flexibility. Mol Cell Neurosci 37: 236-246.

Fatemi SH (2005). Neuropsychiatric Disorders and Infection. Martin Dunitz-Taylor \& Francis Group: London, UK.

Fatemi SH, Folsom TD (2009). The neurodevelopmental hypothesis of schizophrenia, revisited. Schizophr Bull 35: 528-548.

Fatemi SH, Reutiman TJ, Folsom TD, Huang H, Oishi K, Mori S et al (2008). Maternal infection leads to abnormal gene regulation and brain atrophy in mouse offspring: implications for genesis of neurodevelopmental disorders. Schizophr Res 99: 56-70.

Fernandez Espejo E (2003). Prefrontocortical dopamine loss in rats delays long-term extinction of contextual conditioned fear, and reduces social interaction without affecting short-term social interaction memory. Neuropsychopharmacology 28: 490-498.

Flor-Henry P (1990). Influence of gender in schizophrenia as related to other psychopathological syndromes. Schizophr Bull 16: $211-227$

Foussias G, Remington G (2010). Negative symptoms in schizophrenia: Avolition and Occam's razor. Schizophr Bull 36: 359-369.

Gaisler-Salomon I, Diamant L, Rubin C, Weiner I (2008). Abnormally persistent latent inhibition induced by MK801 is reversed by risperidone and by positive modulators of NMDA receptor function: differential efficacy depending on the stage of the task at which they are administered. Psychopharmacology 196: $255-267$.

Gaisler-Salomon I, Weiner I (2003). Systemic administration of MK-801 produces an abnormally persistent latent inhibition which is reversed by clozapine but not haloperidol. Psychopharmacology 166: 333-342.

Goff DC, Coyle JT (2001). The emerging role of glutamate in the pathophysiology and treatment of schizophrenia. Am J Psychiatry 158: 1367-1377.

Hava G, Vered L, Yael M, Mordechai H, Mahoud H (2006). Alterations in behavior in adult offspring mice following maternal inflammation during pregnancy. Dev Psychobiol 48: $162-168$.

Hayward MD, Schaich-Borg A, Pintar JE, Low MJ (2006). Differential involvement of endogenous opioids in sucrose consumption and food reinforcement. Pharmacol Biochem Behav 85: 601-611.

Hirschowitz J, Zemlan FP, Hitzemann RJ, Fleischmann RL, Garver DL (1986). Growth hormone response to apomorphine and diagnosis: a comparison of three diagnostic systems. Biol Psychiatry 21: 445-454.

Horan WP, Blanchard JJ, Clark LA, Green MF (2008). Affective traits in schizophrenia and schizotypy. Schizophr Bull 34: 856-874.

Juckel G, Sass L, Heinz A (2003). Anhedonia, self-experience in schizophrenia, and implications for treatment. Pharmacopsychiatry 36: S176-S180.

Kimura M, Toth LA, Agostini H, Cady AB, Majde JA, Krueger JM (1994). Comparison of acute phase responses induced in rabbits by lipopolysaccharide and double-stranded RNA. Am J Physiol 267: R1596-R1605.
Knable MB, Weinberger DR (1997). Dopamine, the prefrontal cortex and schizophrenia. J Psychopharmacol 11: 123-131.

Koenig JI, Kirkpatrick B, Lee P (2002). Glucocorticoid hormones and early brain development in schizophrenia. Neuropsychopharmacology 27: 309-318.

Li Q, Cheung C, Wei R, Hui ES, Feldon J, Meyer U et al (2009). Prenatal immune challenge is an environmental risk factor for brain and behavior change relevant to schizophrenia: evidence from MRI in a mouse model. PLoS One 4: e6354.

Lipina T, Labrie V, Weiner I, Roder J (2005). Modulators of the glycine site on NMDA receptors, D-serine and ALX 5407, display similar beneficial effects to clozapine in mouse models of schizophrenia. Psychopharmacology 179: 54-67.

Lubow RE (2005). Construct validity of the animal latent inhibition model of selective attention deficits in schizophrenia. Schizophr Bull 31: 139-153.

McGlashan TH, Fenton WS (1992). The positive-negative distinction in schizophrenia. Review of natural history validators. Arch Gen Psychiatry 49: 63-72.

Meltzer HY, Kolakowska T, Fang VS, Fogg L, Robertson A, Lewine $\mathrm{R}$ et al (1984). Growth hormone and prolactin response to apomorphine in schizophrenia and the major affective disorders. Relation to duration of illness and depressive symptoms. Arch Gen Psychiatry 41: 512-519.

Meyer U, Feldon J (2009). Neural basis of psychosis-related behaviour in the infection model of schizophrenia. Behav Brain Res 204: 322-334.

Meyer U, Feldon J (2010). Epidemiology-driven neurodevelopmental animal models of schizophrenia. Prog Neurobiol 90: 285-326.

Meyer U, Feldon J, Fatemi SH (2009a). In-vivo rodent models for the experimental investigation of prenatal immune activation effects in neurodevelopmental brain disorders. Neurosci Biobehav Rev 33: 1061-1079.

Meyer U, Feldon J, Schedlowski M, Yee BK (2005). Towards an immuno-precipitated neurodevelopmental animal model of schizophrenia. Neurosci Biobehav Rev 29: 913-947.

Meyer U, Feldon J, Schedlowski M, Yee BK (2006a). Immunological stress at the maternal-foetal interface: a link between neurodevelopment and adult psychopathology. Brain Behav Immun 20: 378-388.

Meyer U, Feldon J, Yee BK (2009b). A review of the fetal brain cytokine imbalance hypothesis of schizophrenia. Schizophr Bull 35: 959-972.

Meyer U, Knuesel I, Nyffeler M, Feldon J (2010). Chronic clozapine treatment improves prenatal infection-induced working memory deficits without influencing adult hippocampal neurogenesis. Psychopharmacology 208: 531-543.

Meyer U, Murray PJ, Urwyler A, Yee BK, Schedlowski M, Feldon J (2008a). Adult behavioral and pharmacological dysfunctions following disruption of the fetal brain balance between proinflammatory and IL-10-mediated anti-inflammatory signaling. Mol Psychiatry 13: 208-221.

Meyer U, Nyffeler M, Engler A, Urwyler A, Schedlowski M, Knuesel I et al (2006b). The time of prenatal immune challenge determines the specificity of inflammation-mediated brain and behavioral pathology. J Neurosci 26: 4752-4762.

Meyer U, Nyffeler M, Schwendener S, Knuesel I, Yee BK, Feldon J (2008b). Relative prenatal and postnatal maternal contributions to schizophrenia-related neurochemical dysfunction after in utero immune challenge. Neuropsychopharmacology 33: 441-456.

Meyer U, Nyffeler M, Yee BK, Knuesel I, Feldon J (2008c). Adult brain and behavioral pathological markers of prenatal immune challenge during early/middle and late fetal development in mice. Brain Behav Immun 22: 469-486.

Meyer U, Schwendener S, Feldon J, Yee BK (2006c). Prenatal and postnatal maternal contributions in the infection model of schizophrenia. Exp Brain Res 173: 243-257. 
Meyer U, Yee BK, Feldon J (2007). The neurodevelopmental impact of prenatal infections at different times of pregnancy: the earlier the worse? Neuroscientist 13: 241-256.

Morrens M, Hulstijn W, Lewi PJ, De Hert M, Sabbe BG (2006). Stereotypy in schizophrenia. Schizophr Res 84: 397-404.

Ozawa K, Hashimoto K, Kishimoto T, Shimizu E, Ishikura H, Iyo $M$ (2006). Immune activation during pregnancy in mice leads to dopaminergic hyperfunction and cognitive impairment in the offspring: a neurodevelopmental animal model of schizophrenia. Biol Psychiatry 59: 546-554.

Patterson PH (2007). Maternal effects on schizophrenia risk. Science 318: 576-577.

Patterson PH (2009). Immune involvement in schizophrenia and autism: etiology, pathology and animal models. Behav Brain Res 204: 313-321.

Peleg-Raibstein D, Knuesel I, Feldon J (2008). Amphetamine sensitization in rats as an animal model of schizophrenia. Behav Brain Res 191: 190-201.

Peleg-Raibstein D, Pezze MA, Ferger B, Zhang WN, Murphy CA, Feldon J et al (2005). Activation of dopaminergic neurotransmission in the medial prefrontal cortex by $N$-methyl-D-aspartate stimulation of the ventral hippocampus in rats. Neuroscience 132: 219-232.

Pothion S, Bizot JC, Trovero F, Belzung C (2004). Strain differences in sucrose preference and in the consequences of unpredictable chronic mild stress. Behav Brain Res 155: 135-146.

Protais P, Costentin J, Schwartz JC (1976). Climbing behavior induced by apomorphine in mice: a simple test for the study of dopamine receptors in striatum. Psychopharmacology 50: 1-6.

Rapoport JL, Addington AM, Frangou S, Psych MR (2005). The neurodevelopmental model of schizophrenia: update 2005. Mol Psychiatry 10: 434-449.

Rascle C, Mazas O, Vaiva G, Tournant M, Raybois O, Goudemand $\mathrm{M}$ et al (2001). Clinical features of latent inhibition in schizophrenia. Schizophr Res 51: 149-161.

Remington G, Foussias G, Agid O (2010). Progress in defining optimal treatment outcome in schizophrenia. CNS Drugs 24: 9-20.

Roy MA, Maziade M, Labbé A, Mérette C (2001). Male gender is associated with deficit schizophrenia: a meta-analysis. Schizophr Res 47: 141-147.

Schooler C, Spohn HE (1982). Social dysfunction and treatment failure in schizophrenia. Schizophr Bull 8: 85-98.

Shi L, Fatemi SH, Sidwell RW, Patterson PH (2003). Maternal influenza infection causes marked behavioral and pharmacological changes in the offspring. J Neurosci 23: 297-302.

Singer P, Boison D, Möhler H, Feldon J, Yee BK (2009). Deletion of glycine transporter 1 (GlyT1) in forebrain neurons facilitates reversal learning: enhanced cognitive adaptability? Behav Neurosci 123: 1012-1027.

Slattery DA, Markou A, Cryan JF (2007). Evaluation of reward processes in an animal model of depression. Psychopharmacology 190: 555-568.

Smith SE, Li J, Garbett K, Mirnics K, Patterson PH (2007). Maternal immune activation alters fetal brain development through interleukin-6. J Neurosci 27: 10695-10702.

Sullivan R, Wilson DA, Feldon J, Yee BK, Meyer U, Richter-Levin G et al (2006). The International Society for Developmental Psychobiology annual meeting symposium: impact of early life experiences on brain and behavioral development. Dev Psychobiol 48: 583-602.
Susser E, St Clair D, He L (2008). Latent effects of prenatal malnutrition on adult health: the example of schizophrenia. Ann N Y Acad Sci 1136: 185-192.

Tamminga CA (2006). The neurobiology of cognition in schizophrenia. J Clin Psychiatry 67(Suppl 9): 9-13.

Tirelli E, Witkin JM (1994). Transient hypersensitivity to apomorphine-induced gnawing after termination of acute effects of a single high dose of cocaine. Behav Pharmacol 5: 289-298.

Traynor TR, Majde JA, Bohnet SG, Krueger JM (2004). Intratracheal double-stranded RNA plus interferon-gamma: a model for analysis of the acute phase response to respiratory viral infections. Life Sci 74: 2563-2576.

Vuillermot S, Weber L, Feldon J, Meyer U (2010). A longitudinal examination of the neurodevelopmental impact of prenatal immune activation in mice reveals primary defects in dopaminergic development relevant to schizophrenia. J Neurosci 30: 1270-1287.

Watson CG, Kucala T, Angulski G, Vassar P (1987). The relationships of anhedonia and the process-reactive dimension to season of birth and infectious disease incidence in schizophrenia. J Nerv Ment Dis 175: 34-40.

Weinberger DR (1987). Implications of normal brain development for the pathogenesis of schizophrenia. Arch Gen Psychiatry 44: 660-669.

Weiner I (1990). Neural substrates of latent inhibition: the switching model. Psychol Bull 108: 442-461.

Weiner I (2003). The 'two-headed' latent inhibition model of schizophrenia: modeling positive and negative symptoms and their treatment. Psychopharmacology 169: 257-297.

Weiner I, Arad M (2009). Using the pharmacology of latent inhibition to model domains of pathology in schizophrenia and their treatment. Behav Brain Res 204: 369-386.

Winter C, Djodari-Irani A, Sohr R, Morgenstern R, Feldon J, Juckel G et al (2009). Prenatal immune activation leads to multiple changes in basal neurotransmitter levels in the adult brain: implications for brain disorders of neurodevelopmental origin such as schizophrenia. Int J Neuropsychopharmacol 12: 513-524.

Winter C, Reutiman TJ, Folsom TD, Sohr R, Wolf RJ, Juckel G et al (2008). Dopamine and serotonin levels following prenatal viral infection in mouse - implications for psychiatric disorders such as schizophrenia and autism. Eur Neuropsychopharmacol 18: 712-716.

Wise RA (2008). Dopamine and reward: the anhedonia hypothesis 30 years on. Neurotox Res 14: 169-183.

Wolff AR, Bilkey DK (2008). Immune activation during midgestation disrupts sensorimotor gating in rat offspring. Behav Brain Res 190: 156-159.

Zemlan FP, Hirschowitz J, Garver DL (1986). Relation of clinical symptoms to apomorphine-stimulated growth hormone release in mood-incongruent psychotic patients. Arch Gen Psychiatry 43: $1162-1167$.

Zuckerman L, Rehavi M, Nachman R, Weiner I (2003). Immune activation during pregnancy in rats leads to a postpubertal emergence of disrupted latent inhibition, dopaminergic hyperfunction, and altered limbic morphology in the offspring: a novel neurodevelopmental model of schizophrenia. Neuropsychopharmacology 28: 1778-1789.

Zuckerman L, Weiner I (2005). Maternal immune activation leads to behavioral and pharmacological changes in the adult offspring. J Psychiatr Res 39: 311-323.

Supplementary Information accompanies the paper on the Neuropsychopharmacology website (http://www.nature.com/npp) 